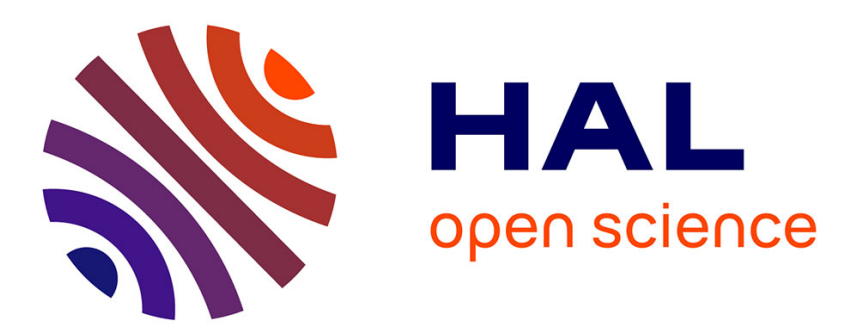

\title{
An optimal condition of compactness for elasticity problems involving one directional reinforcement \\ Marc Briane, Mohamed Camar-Eddine
}

\section{To cite this version:}

Marc Briane, Mohamed Camar-Eddine. An optimal condition of compactness for elasticity problems involving one directional reinforcement. Journal of Elasticity, 2012, 107 (1), pp.11-38. 10.1007/s10659-011-9334-5 . hal-00497567

\section{HAL Id: hal-00497567 https://hal.science/hal-00497567}

Submitted on 5 Jul 2010

HAL is a multi-disciplinary open access archive for the deposit and dissemination of scientific research documents, whether they are published or not. The documents may come from teaching and research institutions in France or abroad, or from public or private research centers.
L'archive ouverte pluridisciplinaire HAL, est destinée au dépôt et à la diffusion de documents scientifiques de niveau recherche, publiés ou non, émanant des établissements d'enseignement et de recherche français ou étrangers, des laboratoires publics ou privés. 


\title{
An optimal condition of compactness for elasticity problems involving one directional reinforcement
}

\author{
M. Briane And M. Camar-Eddine
}

July 2, 2010

\begin{abstract}
This paper deals with the homogenization of a homogeneous elastic medium reinforced by very stiff strips in dimension two. We give a general condition linked to the distribution and the stiffness of the strips, under which the nature of the elasticity problem is preserved in the homogenization process. This condition is sharper than the one used in [1] and is shown to be optimal in the case where the strips are periodically arranged. Indeed, a fourth-order derivative term appears in the limit equation as soon as the condition is no more satisfied. In the periodic case the influence of oscillations in the medium surrounding the strips is also considered. The homogenization method is based both on a two-scale convergence for the strips and the use of suitable oscillating test functions. This allows us to obtain a distributional convergence of two of the three entries of the stress tensor contrary to the $\Gamma$-convergence approach of [11].
\end{abstract}

keywords : Homogenization; H-convergence; two-scale convergence; oscillating test functions method; elasticity

\section{Introduction}

In this paper we are interested in the asymptotic behaviour of two-dimensional linear elasticity equations with equi-coercive but non uniformly bounded tensors $\boldsymbol{A}^{\varepsilon}$,

$$
\left\{\begin{aligned}
-\operatorname{Div}\left(\boldsymbol{A}^{\varepsilon} \boldsymbol{e}\left(\boldsymbol{u}^{\varepsilon}\right)\right) & =\boldsymbol{f} \text { in } \Omega \\
\boldsymbol{u}^{\varepsilon} & =\mathbf{0} \text { on } \partial \Omega,
\end{aligned}\right.
$$

where $\Omega$ is a bounded connected open subset of $\mathbb{R}^{2}$ with a Lipschitz boundary and $\boldsymbol{f}$ is an element of $H^{-1}\left(\Omega, \mathbb{R}^{2}\right)$. The knowledge of the limits of such equations is of great interest in the homogenization theory. In order to place the paper in context, let us review a few of the previous works in the topic of homogenization of elliptic equations with high-contrast parameters.

In the conduction setting, when the coefficients are uniformly bounded, Spagnolo [26] with the $G$-convergence theory, Murat \& Tartar [24, 27] with the $H$-convergence theory, adapted to the elasticity setting by Francfort \& Murat [19], proved the compactness of the class of equations of the type (2.6). More precisely, they showed that the solution $u_{\varepsilon} \in \mathrm{H}_{0}^{1}(\Omega)$ of the conduction problem

$$
-\operatorname{div}\left(A^{\varepsilon} \nabla u_{\varepsilon}\right)=f \quad \text { in } \quad \mathcal{D}^{\prime}(\Omega),
$$

with uniformly bounded conductivity $A^{\varepsilon}$, strongly converges in $\mathrm{L}^{2}(\Omega)$, up to a subsequence of $\varepsilon$, to the solution of a limit conduction problem of the same nature. Buttazzo \& Dal 
Maso [15] extended this compactness result to sequences of isotropic conductivities which are only bounded and equi-integrable in $\mathrm{L}^{1}(\Omega)$. In the periodic framework a refinement of these compactness results is given in [9].

When the coefficients are not assumed to be uniformly bounded, it is well known that the limit equation of $(1.2)$ may be dramatically different from the original one. Khruslov [18, 20, 21] was one of the first authors to obtain different types of limit behaviours such as nonlocal and memory effects in the context of highly contrasted conductivities. His results have been extended by many authors in various directions (see, for instance, [22, 7, 12, 14, 16, 8, 9]). But, in conduction, the class of limit behaviours is well identified. Indeed, Mosco [22] proved that the limit energy associated with the homogenized problem satisfies the BeurlingDeny representation formula [6] of the Dirichlet forms. Conversely, Seppecher and the second author [16] showed that, in dimension greater than two, any Dirichlet form is the limit of some isotropic diffusion functionals. Non classical limits such as second gradient terms [25] and nonlocal effects [3, 4] were also observed in elasticity, mainly by the means of the fiber-reinforcement principle first used by Khruslov in conduction to derive non-local effects. In dimension greater than two, Seppecher and the second author [17] showed that any lower semicontinuous and objective (i.e., vanishing for rigid motions) quadratic functional of displacements is the limit of some sequence of isotropic elastic energies.

Most of the non classical behaviours and closure results we described above hold true in dimension greater than two. Indeed, in the two-dimensional conductivity case Casado-Díaz and the first author [10, 12, 13] proved that the class of equations (1.2) is always compact in the sense that the limit equation of $(1.2)$ is always of the same type. In [10, 12 they proved some extensions of the classical div-curl lemma of Murat-Tartar [23] and deduce an extension of the H-convergence in dimension two.

Under the following control on the sequence of elasticity tensors $\boldsymbol{A}^{\varepsilon}$

$$
\left\{\begin{aligned}
\left\|\boldsymbol{A}^{\varepsilon}\right\|_{\mathrm{L}^{1}} \text { is bounded, } & \text { in the non-periodic case } \\
\varepsilon^{2}\left\|\boldsymbol{A}^{\varepsilon}\right\|_{\mathrm{L}^{1}} \underset{\varepsilon \rightarrow 0}{\longrightarrow} 0, & \text { in the periodic case, }
\end{aligned}\right.
$$

it is proved in [11] that the homogenization of the elasticity problem is similar to the conductivity one since the div-curl approach of [10, 12, applies. Moreover, in the periodic setting, the counter-example in [1] satisfying the asymptotic

$$
\varepsilon^{2}\left\|\boldsymbol{A}^{\varepsilon}\right\|_{\mathrm{L}^{1}} \underset{\varepsilon \rightarrow 0}{\longrightarrow} \infty
$$

shows that contrary to the scalar case of [13], strong stiffness may induce a degenerate limit behaviour. In view of the gap between the second condition of $(1.3)$ and $(1.4)$, the analysis of [11] does not provide a critical barrier below which the nature of the elasticity problem is preserved and above which a degeneracy may appear.

The aim of this paper is to determine such a barrier in the case of an elastic medium reinforced by very stiff strips in the $x_{2}$-direction. Assuming that the medium is homogeneous but the distribution $F_{\varepsilon}$ of the strips is quite general, we introduce a sequence $\psi_{\varepsilon}$ of functions depending only on the $x_{1}$ variable, which strongly converges to zero in $\mathrm{H}^{1}$ such that $\nabla \psi_{\varepsilon}=\boldsymbol{e}_{1}$ in $F_{\varepsilon}$. Then, the desired barrier is characterized by the limit

$$
b:=\lim _{\varepsilon \rightarrow 0} \int_{F_{\varepsilon}} \psi_{\varepsilon}^{2}\left\|\boldsymbol{A}^{\varepsilon}\right\| d x
$$

where $\boldsymbol{A}^{\varepsilon}$ is the elasticity tensor. 
First, if $b=0$ then we prove a compactness result (Theorem 1), in the sense that the nature of the elasticity problem is preserved through the homogenization process. Otherwise, considering a general periodic distribution of strips we prove that the case $b>0$ induces a fourth-order derivative term in the limit problem (Theorem 2). These two results thus show that the limit $b$ measures the appearance of a degeneracy. Moreover, it is easy to check that when $\boldsymbol{A}^{\varepsilon}$ is $\varepsilon$-periodic, the condition $b=0$ is sharper than the second condition of $(1.3)$ and can even be satisfied together with (1.4). The key ingredient of the proof is a two-scale convergence approach combined with the construction of a sequence $\psi_{\varepsilon}$ associated with the distribution of the strips in the surrounding medium.

On the other hand, we extend the previous result to the case where the medium has an oscillating elastic tensor which is uniformly bounded from below and above. The use of appropriate oscillating test functions allows us to obtain the distributional limits of the entries $\boldsymbol{\xi}_{11}^{\varepsilon}$ and $\boldsymbol{\xi}_{12}^{\varepsilon}$ (but not $\boldsymbol{\xi}_{22}^{\varepsilon}$ ) of the stress tensor $\boldsymbol{\xi}^{\varepsilon}=\boldsymbol{A}^{\varepsilon} \boldsymbol{e}\left(\boldsymbol{u}^{\varepsilon}\right)$. The proof is rather delicate since the two sequences are not necessarily bounded in $\mathrm{L}^{1}$ due to the stiffness condition (1.4).

The paper is organized as follows. In Section 2 we give some general notations and state the main results. Section 3 is devoted to the proofs of the results. We first prove the compactness result of Theorem 1 under the sufficient condition (2.13) for non-periodic coefficients. Then, we proceed with the proof of Theorem 2 on the optimality of condition 2.13 in the periodic setting. Finally, we prove Theorem 3 on the influence of the oscillations in the surrounding medium.

\section{Main results}

We start this section by giving some general notations used throughout the paper.

\subsection{General notations and definitions}

- $\Omega$ is a bounded connected open subset of $\mathbb{R}^{2}$ with a Lipschitz boundary. The unit square $(0,1)^{2}$ of $\mathbb{R}^{2}$ is denoted by $Y$.

- For any subset $\omega$ of $\Omega$, we denote by $\bar{\omega}$ the closure of $\omega$ in $\mathbb{R}^{2}$.

- $\varepsilon$ is a sequence of positive real numbers.

- The space of $(2 \times 2)$ real-valued symmetric matrices is denoted by $\mathbb{R}_{s}^{2 \times 2}$. The identity matrix of $\mathbb{R}_{s}^{2 \times 2}$ is denoted by $\boldsymbol{I}_{2}$, while the identity fourth-order tensor is denoted by $\boldsymbol{I}_{4}$.

- The scalar product of two vectors $\boldsymbol{u}$ and $\boldsymbol{v}$ of $\mathbb{R}^{2}$ is denoted by $\boldsymbol{u} \cdot \boldsymbol{v}$, and the one of two matrices $\boldsymbol{\sigma}, \boldsymbol{\xi} \in \mathbb{R}^{2 \times 2}$ is denoted by

$$
\boldsymbol{\sigma}: \boldsymbol{\xi}=\operatorname{Tr}\left(\boldsymbol{\sigma}^{t} \boldsymbol{\xi}\right)
$$

where $\boldsymbol{\sigma}^{t}$ is the transpose of $\boldsymbol{\sigma}$ and $\operatorname{Tr}(\boldsymbol{\sigma})$ its trace. We denote the norm of a vector $\boldsymbol{u} \in \mathbb{R}^{2}$ by $|\boldsymbol{u}|$ and the one of a matrix $\boldsymbol{\sigma} \in \mathbb{R}^{2 \times 2}$ by $\|\boldsymbol{\sigma}\|$.

- For any $\boldsymbol{u}$ and $\boldsymbol{v}$ in $\mathbb{R}^{2}$ we denote by $\boldsymbol{u} \otimes \boldsymbol{v}$ and $\boldsymbol{u} \odot \boldsymbol{v}$ the $(2 \times 2)$-matrices the components of which are defined by

$$
(\boldsymbol{u} \otimes \boldsymbol{v})_{i j}:=u_{i} v_{j} \quad \text { and } \quad \boldsymbol{u} \odot \boldsymbol{v}:=\frac{1}{2}(\boldsymbol{u} \otimes \boldsymbol{v}+\boldsymbol{v} \otimes \boldsymbol{u}) .
$$


- For any $\boldsymbol{\sigma}$ and $\boldsymbol{\xi}$ in $\mathbb{R}_{s}^{2 \times 2}$ we denote by $\boldsymbol{\sigma} \otimes \boldsymbol{\xi}$ the fourth-order tensor the components of which are defined by

$$
(\boldsymbol{\sigma} \otimes \boldsymbol{\xi})_{i j k l}:=\sigma_{i j} \xi_{k l} .
$$

- The gradient of a displacement $\boldsymbol{u} \in \mathbb{R}^{2}$ is the $(2 \times 2)$ matrix $\nabla \boldsymbol{u}$ the entries of which are defined by

$$
(\nabla \boldsymbol{u})_{i j}:=\frac{\partial u_{i}}{\partial x_{j}}
$$

The divergence of a matrix $\boldsymbol{\sigma}$ is the vector $\operatorname{Div}(\boldsymbol{\sigma})$ the components of which are defined by

$$
(\operatorname{Div}(\boldsymbol{\sigma}))_{i}:=\frac{\partial \sigma_{i j}}{\partial x_{j}},
$$

where the Einstein summation convention over repeated indices is used.

- The symmetric part of the gradient of a displacement $\boldsymbol{u}$ is denoted by $\boldsymbol{e}(\boldsymbol{u}) i$.e.,

$$
\boldsymbol{e}(\boldsymbol{u}):=\frac{1}{2}\left(\nabla \boldsymbol{u}+(\nabla \boldsymbol{u})^{t}\right)
$$

Note that, for any symmetric fourth-order tensor $\boldsymbol{A}$, we have $\boldsymbol{A} \boldsymbol{e}(\boldsymbol{u})=\boldsymbol{A} \nabla \boldsymbol{u}$. Therefore, we will use indifferently both expressions.

- The support of a function $\varphi$ is denoted by $\operatorname{supp} \varphi$. The space of infinitely differentiable functions with compact support in $\Omega$ is denoted by $\mathcal{D}(\Omega)$.

- We denote by $C_{0}(\Omega)$ the space of continuous functions on $\bar{\Omega}$ vanishing on the boundary $\partial \Omega$ of $\Omega$, and by $\mathcal{M}(\Omega)$ the set of Radon measures on $\Omega$. A sequence $\left(\mu_{\varepsilon}\right)$ in $\mathcal{M}(\Omega)$ is said to weakly $*$ converge to a measure $\mu$ if

$$
\int_{\Omega} \varphi \mu_{\varepsilon}(d x) \underset{\varepsilon \rightarrow 0}{\longrightarrow} \int_{\Omega} \varphi \mu(d x), \quad \text { for any } \varphi \in C_{0}(\Omega) .
$$

- We denote by $\mathrm{H}^{1}\left(\Omega, \mathbb{R}^{2}\right)$ the usual Sobolev space, endowed with its standard norm

$$
\|\boldsymbol{u}\|_{\mathrm{H}^{1}\left(\Omega, \mathbb{R}^{2}\right)}:=\left(\int_{\Omega}|\boldsymbol{u}(x)|^{2} d x+\int_{\Omega}|\nabla \boldsymbol{u}(x)|^{2} d x\right)^{1 / 2}
$$

- The space of $Y$-periodic functions which belong to $\mathrm{L}_{\text {loc }}^{p}\left(\mathbb{R}^{2}\right)$ (resp. $\left.\mathrm{H}_{\text {loc }}^{1}\left(\mathbb{R}^{2}\right)\right)$ is denoted by $\mathrm{L}_{\#}^{p}(Y)\left(\right.$ resp. $\left.\mathrm{H}_{\#}^{1}(Y)\right)$.

- We denote by $|\omega|$ the Lebesgue measure of any Borel subset $\omega \subset \Omega$, and by

$$
f_{\omega} u d x:=\frac{1}{|\omega|} \int_{\omega} u d x
$$

the average-value of any function $u \in \mathrm{L}^{1}(\omega)$.

- We denote by $\mathbf{1}_{\omega}$ the characteristic function of the set $\omega$.

- $O(\varepsilon)$ denotes a term bounded by a constant times $\varepsilon$ while $o(\varepsilon)$ denotes a term of the form $\varepsilon \theta(\varepsilon)$ where the limit of $\theta(\varepsilon)$ is zero, as $\varepsilon$ goes to zero. 
- Throughout the paper, the letter $c$ denotes a positive constant whose value is not given explicitly and that may vary from line to line.

Let $\left(\boldsymbol{A}^{\varepsilon}\right)$ be a sequence of symmetric fourth-order tensor-valued functions satisfying

$$
\boldsymbol{A}^{\varepsilon}(x) \boldsymbol{\xi}: \boldsymbol{\xi} \geq \alpha\|\boldsymbol{\xi}\|^{2}, \quad \text { a.e. } x \in \Omega, \forall \boldsymbol{\xi} \in \mathbb{R}_{s}^{2 \times 2},
$$

for some positive constant $\alpha$. In the particular case of isotropic elastic materials, the tensor $\boldsymbol{A}^{\varepsilon}$ is determined by the Lamé coefficients $\left(\lambda_{\varepsilon}, \mu_{\varepsilon}\right)$ as follows:

$$
\boldsymbol{A}^{\varepsilon}(x)=2 \mu_{\varepsilon}(x) \boldsymbol{I}_{4}+\lambda_{\varepsilon}(x) \boldsymbol{I}_{2} \otimes \boldsymbol{I}_{2} \quad \text { a.e. } x \in \Omega,
$$

or equivalently,

$$
\boldsymbol{A}^{\varepsilon}(x) \boldsymbol{\xi}=2 \mu_{\varepsilon}(x) \boldsymbol{\xi}+\lambda_{\varepsilon}(x) \operatorname{Tr}(\boldsymbol{\xi}) \boldsymbol{I}_{2} \quad \text { a.e. } x \in \Omega, \quad \forall \boldsymbol{\xi} \in \mathbb{R}_{s}^{2 \times 2} .
$$

One can check that

$$
\boldsymbol{A}^{\varepsilon}(x) \boldsymbol{\xi}: \boldsymbol{\xi} \leq 2 \max \left(\mu_{\varepsilon}(x), \lambda_{\varepsilon}(x)+\mu_{\varepsilon}(x)\right)\|\boldsymbol{\xi}\|^{2}, \quad \text { a.e. } x \in \Omega, \quad \forall \boldsymbol{\xi} \in \mathbb{R}_{s}^{2 \times 2},
$$

and that condition $(2.1)$ is equivalent to

$$
0<\alpha \leq 2 \min \left(\mu_{\varepsilon}(x), \lambda_{\varepsilon}(x)+\mu_{\varepsilon}(x)\right) \quad \text { a.e. } x \in \Omega .
$$

Note that in (2.4)-(2.5) the coefficient $\lambda_{\varepsilon}$ is not necessarily non-negative.

Let $\boldsymbol{f}$ be an element of $\mathrm{H}^{-1}\left(\Omega, \mathbb{R}^{2}\right)$. Consider the sequence of elasticity problems

$$
\left\{\begin{array}{rllll}
-\operatorname{Div}\left(\boldsymbol{A}^{\varepsilon} \boldsymbol{e}\left(\boldsymbol{u}^{\varepsilon}\right)\right) & = & \boldsymbol{f} & \text { in } & \Omega \\
\boldsymbol{u}^{\varepsilon} & = & \mathbf{0} & \text { on } & \partial \Omega .
\end{array}\right.
$$

We are interested in finding a condition which preserves the nature of the elasticity equation (2.6). For the sake of simplicity, this preservation property will be called the compactness of the equations $(2.6)$.

\subsection{Sufficient conditions for compactness}

For homogeneous media reinforced with parallel and very stiff strips the Lamé coefficients of which are unbounded we give an estimate of the stress tensor $\boldsymbol{\xi}^{\varepsilon}:=\boldsymbol{A}^{\varepsilon} \boldsymbol{e}\left(\boldsymbol{u}^{\varepsilon}\right)$ and provide a sufficient condition which ensures the compactness of equations $(2.6)$.

Let $\left(F_{k}^{\varepsilon}\right)$ be a sequence of disjoint strips in $\Omega$ which are parallel to the $x_{2}$-axis. Denote by $F_{\varepsilon}$ the union of the strips $F_{k}^{\varepsilon}$ and by $\Omega_{\varepsilon}$ the complement of $F_{\varepsilon}$ in $\Omega$ :

$$
F_{\varepsilon}:=\bigcup_{k \geq 1} F_{k}^{\varepsilon} \quad \text { and } \quad \Omega_{\varepsilon}:=\Omega \backslash F_{\varepsilon} .
$$

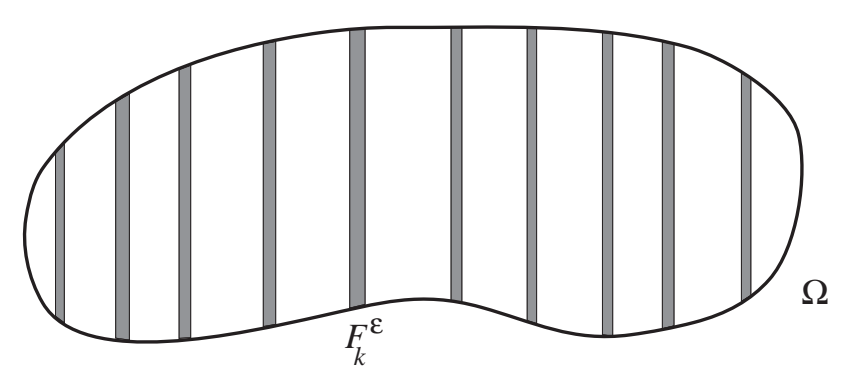

Fig 1. The composite material 
Note that the strips $F_{k}^{\varepsilon}$ are not necessarily periodically distributed in $\Omega$.

Let $\boldsymbol{A}$ be a constant symmetric fourth-order tensor and $\boldsymbol{B}_{\varepsilon}$ be an isotropic symmetric fourthorder tensor-valued function:

$$
\boldsymbol{B}^{\varepsilon}(x)=2 \mu_{\varepsilon}(x) \boldsymbol{I}_{4}+\lambda_{\varepsilon}(x) \boldsymbol{I}_{2} \otimes \boldsymbol{I}_{2} \quad \text { a.e. } x \in \Omega,
$$

where $\mu_{\varepsilon}>0$ and $\lambda_{\varepsilon}+\mu_{\varepsilon}>0$. Assume $\Omega$ is occupied by a heterogeneous elastic material the elasticity tensor of which writes

$$
\boldsymbol{A}^{\varepsilon}(x):=\boldsymbol{A} \mathbf{1}_{\Omega_{\varepsilon}}(x)+\boldsymbol{B}^{\varepsilon}(x) \mathbf{1}_{F_{\varepsilon}}(x) \text { a.e. } x \in \Omega .
$$

We suppose that the Lamé coefficients $\left(\mu_{\varepsilon}, \lambda_{\varepsilon}\right)$ of the material occupying $F_{\varepsilon}$ depend only on the variable $x_{1}$, that $\mu_{\varepsilon}$ is not $\mathrm{L}^{1}$-bounded i.e.,

$$
\lim _{\varepsilon \rightarrow 0} \int_{0}^{1} \mu_{\varepsilon}\left(x_{1}\right) d x_{1}=\infty
$$

and satisfies the following uniform rigidity condition

$$
\frac{\mu_{\varepsilon}}{\bar{\mu}_{\varepsilon}} \rightarrow \sigma \quad \text { weakly } * \text { in } \mathcal{M}([0,1]), \quad \text { where } \quad \bar{\mu}_{\varepsilon}:=\int_{0}^{1} \mu_{\varepsilon}\left(x_{1}\right) d x_{1},
$$

and $\sigma$ is a Radon measure in $\mathcal{M}([0,1])$ with support $\operatorname{supp}(\sigma)=[0,1]$. Note that in the periodic case

$$
\mu_{\varepsilon}\left(x_{1}\right)=\mu_{\varepsilon}^{\sharp}\left(\frac{x_{1}}{\varepsilon}\right) \quad \text { a.e. } x_{1} \in(0,1),
$$

where $\mu_{\varepsilon}^{\sharp}$ is 1-periodic, and the uniform rigidity condition 2.10 is then satisfied since $\sigma=1$. We finally suppose there exists a sequence $\left(\psi_{\varepsilon}\right)$ of functions in $\mathrm{H}^{1}(\Omega)$ depending only on $x_{1}$ and satisfying

$$
\left\{\begin{array}{rlll}
\psi_{\varepsilon} & \underset{\varepsilon \rightarrow 0}{\longrightarrow} & 0 & \text { strongly in } \mathrm{H}^{1}(\Omega), \\
\nabla \psi_{\varepsilon} & = & \boldsymbol{e}_{1} & \text { in } F_{\varepsilon} .
\end{array}\right.
$$

Assumption 2.11 implies some restrictions on the distribution of the strips $F_{k}^{\varepsilon}$ in $\Omega$. This holds true, for instance, when the strips $F_{k}^{\varepsilon}$ are not too close to each other. An example of such a sequence $\left(\psi_{\varepsilon}\right)$ is provided in Section 2.3 .

Theorem 1 below provides an estimate of the stress tensor $\boldsymbol{\xi}^{\varepsilon}$ :

Theorem 1 Assume that (2.9)-(2.11) hold true. Then, the solution $\boldsymbol{u}^{\varepsilon}$ of the elasticity problem (2.6) weakly converges to some $\boldsymbol{u}=\left(u_{1}, 0\right)$ in $\mathrm{H}_{0}^{1}\left(\Omega, \mathbb{R}^{2}\right)$, and for any $i \in\{1,2\}$, we have the estimate

$$
\int_{\Omega} \boldsymbol{\xi}^{\varepsilon}:\left(\boldsymbol{e}_{1} \odot \boldsymbol{e}_{i}\right) \varphi d x=\int_{\Omega} \boldsymbol{A} \boldsymbol{e}(\boldsymbol{u}):\left(\boldsymbol{e}_{1} \odot \boldsymbol{e}_{i}\right) \varphi d x-\int_{\Omega} \psi_{\varepsilon}(x) \boldsymbol{\xi}^{\varepsilon}:\left(\boldsymbol{e}_{i} \otimes \nabla \varphi\right) d x+o(1),
$$

for any $\varphi \in \mathcal{D}(\Omega)$. If, in addition to 2.9)-2.11, we assume that

$$
b:=\lim _{\varepsilon \rightarrow 0} \int_{\Omega} \psi_{\varepsilon}^{2}(x) \max \left(\mu_{\varepsilon}(x), \lambda_{\varepsilon}(x)+\mu_{\varepsilon}(x)\right) d x=0,
$$

then $u_{1}$ is the solution of the limit problem

$$
\left\{\begin{aligned}
-\operatorname{div}\left(\boldsymbol{A}_{1} \nabla u_{1}\right) & =f_{1} \quad \text { in } \quad \Omega \\
u_{1} & =0 \quad \text { on } \quad \partial \Omega,
\end{aligned}\right.
$$

where $\boldsymbol{A}_{1}$ is the symmetric positive matrix defined by

$$
\boldsymbol{A}_{1} \boldsymbol{v}:=\boldsymbol{A}\left(\boldsymbol{e}_{1} \odot \boldsymbol{v}\right) \boldsymbol{e}_{1}, \quad \forall \boldsymbol{v} \in \mathbb{R}^{2} .
$$


Remark 1 Theorem 1 states that if 2.13 is satisfied then the sequence of elasticity equations (2.6) converges to a limit equation of the same type. Condition 2.13 is clearly a weaker assumption than the $\mathrm{L}^{1}(\Omega)$-boundedness of $\boldsymbol{A}^{\varepsilon}$. Then, Theorem 1 is an extension of the results in [11] to a larger class of $\boldsymbol{A}^{\varepsilon}$. The question we address in the next section is the optimality of the condition (2.13): what happens if 2.13 is not satisfied? We provide an answer to this question in the periodic case. We show that condition 2.13 is optimal in the following sense: if (2.13) is satisfied then we have compactness of the class of equations of the type (2.6), otherwise there is a loss of compactness.

Another novelty of the result of Theorem 1, in comparison with the one in [1], is the identification of the term

$$
\int_{\Omega} \psi_{\varepsilon}(x) \boldsymbol{\xi}^{\varepsilon}:\left(\boldsymbol{e}_{i} \otimes \nabla \varphi\right) d x
$$

in the estimate 2.12 which measures exactly the appearance of degeneracy in the homogenization process, depending on whether (2.13) is satisfied or not.

\subsection{Optimality of condition $(2.13)$ in the periodic case}

In this section we present a class of reinforced periodic materials for which condition (2.13) is not satisfied. More precisely, each material is made of a homogeneous medium reinforced by a periodic lattice of very stiff and very thin strips. In the period cell, contrary to the example of [11], a general distribution of strips is considered. Then, using an adapted two-scale convergence approach we determine the limit of 2.16 which turns out to contain a fourthorder derivative term. Therefore, the term (2.16) with the sequence $\psi_{\varepsilon}$ can be regarded as the compactness default in the homogenization of problems 2.6 in the periodic case.

The class of materials:

For the sake of simplicity, we assume $\Omega$ is the open square $(0,1)^{2}$ of $\mathbb{R}^{2}$. Let $\left(\tau_{k}^{\varepsilon}\right)_{k \in \mathbb{N}^{*}}$ be a sequence in $(0,1)$ and $\left(r_{k}^{\varepsilon}\right)_{k \in \mathbb{N}^{*}}$ be a sequence of positive real numbers. For any $k \geq 1$, set

$$
R_{k}^{\varepsilon}:=\frac{r_{k}^{\varepsilon}}{\varepsilon}, \omega_{k}^{\varepsilon}:=\left(\tau_{k}^{\varepsilon}+R_{k}^{\varepsilon}, \tau_{k+1}^{\varepsilon}-R_{k+1}^{\varepsilon}\right), I_{k}^{\varepsilon}:=\left[\tau_{k}^{\varepsilon}-R_{k}^{\varepsilon}, \tau_{k}^{\varepsilon}+R_{k}^{\varepsilon}\right], \quad \text { and } \quad Q_{k}^{\varepsilon}:=I_{k}^{\varepsilon} \times(0,1) .
$$

The sequence $\left(r_{k}^{\varepsilon}\right)$ is chosen in such a way that

$$
\lim _{\varepsilon \rightarrow 0}\left(\sup _{k \geq 1} R_{k}^{\varepsilon}\right)=0 \quad \text { and } \quad Q_{k}^{\varepsilon} \cap Q_{l}^{\varepsilon}=\emptyset \quad \forall k \neq l .
$$

We denote by $F_{k}^{\varepsilon}$ the $\varepsilon Y$-periodic open subset of $\Omega$ resulting from the repetition of the sets $\varepsilon Q_{k}^{\varepsilon}$ in $\Omega$ :

$$
F_{k}^{\varepsilon}:=\Omega \cap \bigcup_{j \in \mathbb{N}} \varepsilon\left(j+Q_{k}^{\varepsilon}\right)
$$

We define the function $r_{\varepsilon}: \mathbb{R}^{2} \rightarrow(0, \infty)$ by

$$
r_{\varepsilon}(x):= \begin{cases}r_{k}^{\varepsilon} & \text { if } x \in F_{k}^{\varepsilon}, \\ 1 & \text { otherwise }\end{cases}
$$

and the projection function $y_{k}^{\varepsilon}: \mathbb{R}^{2} \rightarrow \mathbb{R}$ by

$$
y_{k}^{\varepsilon}\left(x_{1}, x_{2}\right):=\frac{\left(x_{1}-\varepsilon \operatorname{Int}\left(x_{1} / \varepsilon\right)-\varepsilon \tau_{k}^{\varepsilon}\right)}{r_{k}^{\varepsilon}},
$$


where Int $(\cdot)$ denotes the integer part function. Then we have

$$
F_{k}^{\varepsilon}=\left\{x \in \Omega:\left|y_{k}^{\varepsilon}(x)\right|<1\right\} .
$$

We define the reinforced part of the material to be the union of the subsets $F_{k}^{\varepsilon}$ and we denote it by $F_{\varepsilon}$ :

$$
F_{\varepsilon}:=\bigcup_{k \geq 1} F_{k}^{\varepsilon} .
$$

The remaining part of the domain is denoted by $\Omega_{\varepsilon}:=\Omega \backslash F_{\varepsilon}$ and is supposed to be occupied by an isotropic elastic material with a constant elasticity tensor

$$
\boldsymbol{A}:=2 \mu \boldsymbol{I}_{4}+\lambda \boldsymbol{I}_{2} \otimes \boldsymbol{I}_{2} .
$$

For any $k \geq 1$, we assume that $F_{k}^{\varepsilon}$ is made of an isotropic elastic material the Lamé coefficients of which $\left(\lambda_{k}^{\varepsilon}, \mu_{k}^{\varepsilon}\right)$ are constant and satisfy, for some positive real number $\ell$

$$
\frac{\lambda_{k}^{\varepsilon}}{\mu_{k}^{\varepsilon}}=\ell \quad \text { and } \quad \frac{\mu_{k}^{\varepsilon}\left(r_{k}^{\varepsilon}\right)^{3}}{\varepsilon}=\nu_{k}^{\varepsilon},
$$

where $\left(\nu_{k}^{\varepsilon}\right)_{k \in \mathbb{N}^{*}}$ is a sequence of positive real numbers chosen satisfying

$$
\nu:=\lim _{\varepsilon \rightarrow 0} \sum_{k \geq 1} \nu_{k}^{\varepsilon} \in(0, \infty) .
$$

Taking (2.17) into account, condition (2.21) implies that the Lamé coefficients $\left(\lambda_{k}^{\varepsilon}, \mu_{k}^{\varepsilon}\right)$ are not $\mathrm{L}^{1}(\Omega)$-bounded. Then, the domain $\Omega$ is occupied by the heterogeneous elastic material the elasticity tensor of which writes

$$
\boldsymbol{A}^{\varepsilon}(x):=\boldsymbol{A} \mathbf{1}_{\Omega_{\varepsilon}}(x)+\left(2 \mu_{\varepsilon}(x) \boldsymbol{I}_{4}+\lambda_{\varepsilon}(x) \boldsymbol{I}_{2} \otimes \boldsymbol{I}_{2}\right) \mathbf{1}_{F_{\varepsilon}}(x) \quad \text { a.e. } x \in \Omega,
$$

where

$$
\mu_{\varepsilon}(x):=\sum_{k \geq 1} \mu_{k}^{\varepsilon} \mathbf{1}_{F_{k}^{\varepsilon}}(x) \quad \text { and } \quad \lambda_{\varepsilon}(x):=\sum_{k \geq 1} \lambda_{k}^{\varepsilon} \mathbf{1}_{F_{k}^{\varepsilon}}(x) \quad \text { a.e. } x \in \Omega \text {. }
$$

Remark 2 Note that one can have the situation where, for any $k \geq 1$, the sequence $\left(\nu_{k}^{\varepsilon}\right)_{\varepsilon}$ tends to zero, as $\varepsilon$ goes to zero, although $\nu>0$. This means that the contribution of each single strip is zero while the overall contribution of all the strips is not zero.

Let $y_{\varepsilon}^{\sharp}$ and $\psi_{\varepsilon}^{\sharp}$ be the continuous, 1 -periodic and piece-wise affine functions defined on $\mathbb{R}$, by

$$
y_{\varepsilon}^{\sharp}\left(y_{1}\right):= \begin{cases}\frac{1}{R_{1}^{\varepsilon}-\tau_{1}^{\varepsilon}} y_{1} & \text { if } \quad y_{1} \in\left(0, \tau_{1}^{\varepsilon}-R_{1}^{\varepsilon}\right), \\ \frac{1}{R_{k}^{\varepsilon}}\left(y_{1}-\tau_{k}^{\varepsilon}\right) & \text { if } \quad y_{1} \in\left[\tau_{k}^{\varepsilon}-R_{k}^{\varepsilon}, \tau_{k}^{\varepsilon}+R_{k}^{\varepsilon}\right], \\ \frac{y_{1}-\left(\tau_{k}^{\varepsilon}+R_{k}^{\varepsilon}\right)}{R_{k+1}^{\varepsilon}+R_{k}^{\varepsilon}-\left(\tau_{k+1}^{\varepsilon}-\tau_{k}^{\varepsilon}\right)}+R_{k}^{\varepsilon} & \text { if } \quad y_{1} \in\left(\tau_{k}^{\varepsilon}+R_{k}^{\varepsilon}, \tau_{k+1}^{\varepsilon}-R_{k+1}^{\varepsilon}\right),\end{cases}
$$

and

$$
\psi_{\varepsilon}^{\sharp}\left(y_{1}\right):= \begin{cases}\varepsilon R_{1}^{\varepsilon} y_{\varepsilon}^{\sharp}\left(y_{1}\right) & \text { if } \quad y_{1} \in\left(0, \tau_{1}^{\varepsilon}-R_{1}^{\varepsilon}\right), \\ \varepsilon R_{k}^{\varepsilon} y_{\varepsilon}^{\sharp}\left(y_{1}\right) & \text { if } \quad y_{1} \in I_{k}^{\varepsilon}=\left[\tau_{k}^{\varepsilon}-R_{k}^{\varepsilon}, \tau_{k}^{\varepsilon}+R_{k}^{\varepsilon}\right], \\ \varepsilon\left(R_{k+1}^{\varepsilon}+R_{k}^{\varepsilon}\right) y_{\varepsilon}^{\sharp}\left(y_{1}\right) & \text { if } \quad y_{1} \in \omega_{k}^{\varepsilon}=\left(\tau_{k}^{\varepsilon}+R_{k}^{\varepsilon}, \tau_{k+1}^{\varepsilon}-R_{k+1}^{\varepsilon}\right),\end{cases}
$$


for any $k \in \mathbb{N}^{*}$.

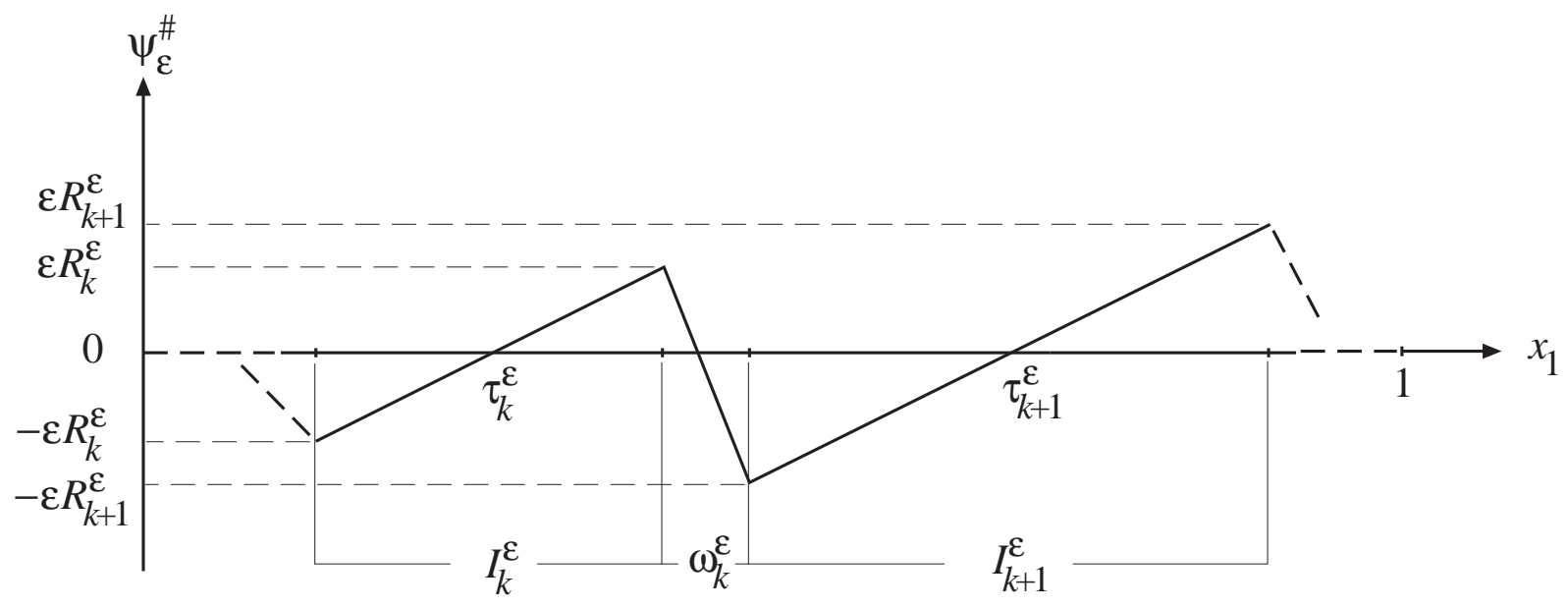

Fig 2. Graph of $\psi_{\varepsilon}^{\sharp}$ on the period cell

Finally, we define the $\varepsilon$-periodic and piece-wise affine functions $y_{\varepsilon}$ and $\psi_{\varepsilon}$ by

$$
y_{\varepsilon}(x):=y_{\varepsilon}^{\sharp}\left(\frac{x_{1}}{\varepsilon}\right) \quad \text { and } \quad \psi_{\varepsilon}(x):=\psi_{\varepsilon}^{\sharp}\left(\frac{x_{1}}{\varepsilon}\right), \quad \text { a.e. } \quad x=\left(x_{1}, x_{2}\right) \in \Omega \text {. }
$$

In view of (2.18), 2.19), 2.24) and 2.25), we have, for any $k \in \mathbb{N}^{*}$

$$
y_{\varepsilon}(x)=y_{k}^{\varepsilon}(x) \text { a.e. } x \in F_{k}^{\varepsilon} \text {, and } \psi_{\varepsilon}(x)=r_{\varepsilon}(x) y_{\varepsilon}(x), \nabla \psi_{\varepsilon}(x)=\boldsymbol{e}_{1} \text { a.e. } x \in F_{\varepsilon} \text {. }
$$

One can check that the sequence $\left(\psi_{\varepsilon}\right)$ defined by $(2.26)$, satisfies the distribution assumption 2.11. However, it satisfies (2.13) if and only if $\nu$, defined by (2.22), is positive. More precisely, the term (2.16) in the estimate 2.12$)$, corresponding to the $\varepsilon Y$-periodic tensor-valued function $\boldsymbol{A}^{\varepsilon}$ defined by $(2.23)$, converges to a second gradient term as the following result shows:

Theorem 2 Assume that (2.17)-(2.22) hold true. Then, for any $\varphi \in \mathcal{D}(\Omega)$ we have

$$
\lim _{\varepsilon \rightarrow 0}\left(\int_{\Omega} \psi_{\varepsilon}(x) \boldsymbol{\xi}^{\varepsilon}:\left(\boldsymbol{e}_{i} \otimes \nabla \varphi\right) d x\right)=\left\{\begin{array}{lll}
0 & \text { if } & i=1, \\
-\eta \int_{\Omega} \frac{\partial^{2} u_{1}}{\partial x_{2}^{2}} \frac{\partial \varphi}{\partial x_{2}}(x) d x & \text { if } \quad i=2,
\end{array}\right.
$$

where $\boldsymbol{u}=\left(u_{1}, 0\right)$ is the weak limit, in $\mathrm{H}^{1}\left(\Omega, \mathbb{R}^{2}\right)$, of the solution $\boldsymbol{u}^{\varepsilon}$ of the elasticity problem (2.6) and

$$
\eta:=\frac{8}{3} \nu\left(\frac{\ell+1}{\ell+2}\right)
$$

Moreover, $u_{1}$ is the unique solution of the fourth-order equation

$$
\left\{\begin{aligned}
-(\lambda+\mu) \frac{\partial^{2} u_{1}}{\partial x_{1}^{2}}-\mu \Delta u_{1}+\eta \frac{\partial^{4} u_{1}}{\partial x_{2}^{4}} & =f_{1} \quad \text { in } \Omega, \\
u_{1} & =0 \quad \text { on } \quad \partial \Omega, \\
\frac{\partial u_{1}}{\partial x_{2}} & =0 \quad \text { on } \quad(0,1) \times\{0,1\} .
\end{aligned}\right.
$$

Remark 3 Theorem 2 implies that the limit equation of (2.6), for the sequence of elasticity tensors $\boldsymbol{A}^{\varepsilon}$ defined by $(2.23)$, is not of the same type as the original one. 


\subsection{Influence of the oscillations in the medium}

In the estimates 2.12 of Theorem 1 the medium surrounding the strips is supposed to be homogeneous with a constant elasticity tensor $\boldsymbol{A}$. In this paragraph we are interested in the case where the medium is isotropic but non homogeneous, and we show the way the homogenized stress tensor is influenced by the oscillations.

Geometry of the problem:

Here, we consider one strip in the period cell. The domain $\Omega$ is the open square $(0,1)^{2}$ of $\mathbb{R}^{2}$. Let $\varepsilon$ and $r_{\varepsilon}$ be sequences of positive real numbers such that $\varepsilon^{-1} \in \mathbb{N}^{*}$ and the sequence $R_{\varepsilon}:=$ $r_{\varepsilon} / \varepsilon$ tends to zero, as $\varepsilon$ goes to zero. We define

$$
\begin{gathered}
I_{\varepsilon}:=\left(1 / 2-R_{\varepsilon}, 1 / 2+R_{\varepsilon}\right), \\
x_{\varepsilon}^{j}:=(j-1 / 2) \varepsilon, \text { for } j \in \mathbb{N}^{*}, \text { and } F_{\varepsilon}:=\Omega \cap \bigcup_{j \in \mathbb{N}^{*}}\left(x_{\varepsilon}^{j}-r_{\varepsilon}, x_{\varepsilon}^{j}+r_{\varepsilon}\right) \times(0,1) .
\end{gathered}
$$

The remaining part of the domain $\Omega$ is denoted by $\Omega_{\varepsilon}:=\Omega \backslash F_{\varepsilon}$.

Let $\lambda_{\varepsilon}^{\sharp}$ and $\mu_{\varepsilon}^{\sharp}$ be two functions defined on $(0,1)$. We assume that $\lambda_{\varepsilon}^{\sharp}$ and $\mu_{\varepsilon}^{\sharp}$ are 1-periodic, bounded from below by 1 on $(0,1)$ and bounded from above in $(0,1) \backslash I_{\varepsilon}$ by a positive constant $c$. But we do not assume that $\lambda_{\varepsilon}^{\sharp}$ and $\mu_{\varepsilon}^{\sharp}$ are uniformly bounded in $(0,1)$. Actually, we make the following assumptions:

$$
\int_{0}^{1} \max \left(\mu_{\varepsilon}^{\sharp}\left(y_{1}\right), \lambda_{\varepsilon}^{\sharp}\left(y_{1}\right)+\mu_{\varepsilon}^{\sharp}\left(y_{1}\right)\right) d y_{1} \underset{\varepsilon \rightarrow 0}{\longrightarrow} \infty
$$

and

$$
\varepsilon^{2}\left(\int_{I_{\varepsilon}} \frac{d y_{1}}{\mu_{\varepsilon}^{\sharp}\left(y_{1}\right)}\right)^{2} \int_{I_{\varepsilon}}\left(\lambda_{\varepsilon}^{\sharp}\left(y_{1}\right)+\mu_{\varepsilon}^{\sharp}\left(y_{1}\right)\right) d y_{1} \underset{\varepsilon \rightarrow 0}{\longrightarrow} 0 .
$$

The technical assumption (2.33) is satisfied, for instance, when there exists a sequence $\alpha_{\varepsilon}$ such that

$$
1 \leq \alpha_{\varepsilon} \leq \lambda_{\varepsilon}^{\sharp}\left(y_{1}\right)+\mu_{\varepsilon}^{\sharp}\left(y_{1}\right) \leq c \alpha_{\varepsilon} \quad \text { a.e. } y_{1} \in I_{\varepsilon},
$$

which corresponds to a rather homogeneous distribution of the values of $\lambda_{\varepsilon}^{\sharp}$ and $\mu_{\varepsilon}^{\sharp}$ in $(0,1)$. Let $\psi_{\varepsilon}^{\sharp}$ be the continuous, 1-periodic and piece-wise affine function defined on $\mathbb{R}$, and satisfying:

$$
\left\{\begin{array}{l}
\left(\psi_{\varepsilon}^{\sharp}\right)^{\prime}\left(y_{1}\right)=\varepsilon \text { if } y_{1} \in I_{\varepsilon}, \\
\psi_{\varepsilon}^{\sharp} \text { is affine elsewhere in }(0,1) .
\end{array}\right.
$$

More precisely,

$$
\psi_{\varepsilon}^{\sharp}\left(y_{1}\right):= \begin{cases}\frac{\varepsilon R_{\varepsilon}}{R_{\varepsilon}-1 / 2} y_{1} & \text { if } \quad y_{1} \in\left(0,1 / 2-R_{\varepsilon}\right), \\ \varepsilon\left(y_{1}-1 / 2\right) & \text { if } \quad y_{1} \in\left[1 / 2-R_{\varepsilon}, 1 / 2+R_{\varepsilon}\right], \\ \frac{\varepsilon R_{\varepsilon}}{R_{\varepsilon}-1 / 2}\left(y_{1}-1\right) & \text { if } \quad y_{1} \in\left(1 / 2+R_{\varepsilon}, 1\right) .\end{cases}
$$




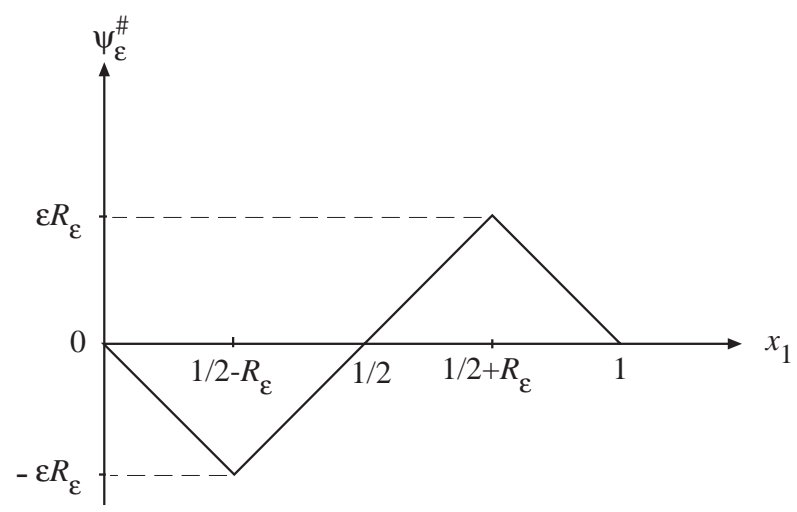

Fig 3. Graph of $\psi_{\varepsilon}^{\sharp}$ on the period cell

Finally, we define the $\varepsilon$-periodic and piece-wise affine function $\psi_{\varepsilon}$ by

$$
\psi_{\varepsilon}(x):=\psi_{\varepsilon}^{\sharp}\left(\frac{x_{1}}{\varepsilon}\right), \quad \text { a.e. } x=\left(x_{1}, x_{2}\right) \in \Omega .
$$

The sequence $\left(\psi_{\varepsilon}\right)$ defined by $(2.36)$ clearly satisfies

$$
\psi_{\varepsilon} \underset{\varepsilon \rightarrow 0}{\longrightarrow} 0 \quad \text { strongly in } \mathrm{H}^{1}(\Omega) \quad \text { and } \quad \nabla \psi_{\varepsilon}(x)=\boldsymbol{e}_{1} \quad \text { a.e. } \quad x \in F_{\varepsilon} .
$$

Let $\left(\boldsymbol{A}_{\varepsilon}^{\sharp}\right)$ be the sequence of $Y$-periodic isotropic fourth-order tensors defined by

$$
\boldsymbol{A}_{\varepsilon}^{\sharp}(y)=2 \mu_{\varepsilon}^{\sharp}\left(y_{1}\right) \boldsymbol{I}_{4}+\lambda_{\varepsilon}^{\sharp}\left(y_{1}\right) \boldsymbol{I}_{2} \otimes \boldsymbol{I}_{2} \quad \text { a.e. } y=\left(y_{1}, y_{2}\right) \in Y .
$$

Let $\boldsymbol{f}$ be an element of $\mathrm{H}^{-1}\left(\Omega, \mathbb{R}^{2}\right)$. Consider the sequence of elasticity problems 2.6 where

$$
\boldsymbol{A}^{\varepsilon}(x):=\boldsymbol{A}_{\varepsilon}^{\sharp}\left(\frac{x}{\varepsilon}\right) \quad \text { a.e. } x \in \Omega \text {. }
$$

Equivalently,

$$
\boldsymbol{A}^{\varepsilon}(x)=2 \mu_{\varepsilon}(x) \boldsymbol{I}_{4}+\lambda_{\varepsilon}(x) \boldsymbol{I}_{2} \otimes \boldsymbol{I}_{2} \quad \text { a.e. } x \in \Omega,
$$

where

$$
\mu_{\varepsilon}(x)=\mu_{\varepsilon}^{\sharp}\left(\frac{x_{1}}{\varepsilon}\right) \quad \text { and } \quad \lambda_{\varepsilon}(x)=\lambda_{\varepsilon}^{\sharp}\left(\frac{x_{1}}{\varepsilon}\right) \quad \text { a.e. } x=\left(x_{1}, x_{2}\right) \in \Omega .
$$

For a fixed $\varepsilon>0$, we denote by $\boldsymbol{A}_{*}^{\varepsilon}$ the constant homogenized tensor induced by the oscillating sequence $\left(\boldsymbol{A}^{\varepsilon}\left(\frac{x}{\delta}\right)\right.$ ), as $\delta$ tends to zero. We recall (see for instance, [5] or [1]) that $\boldsymbol{A}_{*}^{\varepsilon}$ is defined by the minimization problem

$$
\boldsymbol{A}_{*}^{\varepsilon} \boldsymbol{\xi}: \boldsymbol{\xi}=\min \left\{\int_{Y} \boldsymbol{A}_{\varepsilon}^{\sharp}(y)(\boldsymbol{\xi}+\boldsymbol{e}(\boldsymbol{\Psi})):(\boldsymbol{\xi}+\boldsymbol{e}(\boldsymbol{\Psi})) d y: \boldsymbol{\Psi} \in \mathrm{H}_{\#}^{1}\left(Y, \mathbb{R}^{2}\right)\right\},
$$

for any $\boldsymbol{\xi} \in \mathbb{R}_{s}^{2 \times 2}$.

Determination of explicit corrector functions associated with the problem (2.6):

Thanks to the special form of the elasticity tensor $\boldsymbol{A}^{\varepsilon}$ we explicitly compute the corrector functions associated with the problem (2.6). For more details on the role of correctors in the theory of homogenization we refer, for instance, to [5].

Let $\left(\boldsymbol{e}_{1}, \boldsymbol{e}_{2}\right)$ be the canonical basis of $\mathbb{R}^{2}$. It is easy to see that a minimizer in the definition 2.38) of $\boldsymbol{A}_{*}^{\varepsilon}\left(\boldsymbol{e}_{1} \odot \boldsymbol{e}_{1}\right):\left(\boldsymbol{e}_{1} \odot \boldsymbol{e}_{1}\right)$ is the $Y$-periodic function $\boldsymbol{X}^{\varepsilon, 11}$ defined by

$$
\boldsymbol{X}^{\varepsilon, 11}(y):=\left(\int_{\frac{1}{2}}^{y_{1}}\left(1-\frac{c_{\varepsilon}^{1}}{\lambda_{\varepsilon}^{\sharp}+2 \mu_{\varepsilon}^{\sharp}}\right) d t\right) \boldsymbol{e}_{1} \text {, a.e. } y=\left(y_{1}, y_{2}\right) \in(0,1)^{2},
$$


where

$$
c_{\varepsilon}^{1}:=\left(\int_{0}^{1} \frac{d t}{\lambda_{\varepsilon}^{\sharp}+2 \mu_{\varepsilon}^{\sharp}}\right)^{-1} .
$$

We define $\boldsymbol{W}^{\varepsilon, 11}$ by

$$
\boldsymbol{W}^{\varepsilon, 11}(y):=y_{1} \boldsymbol{e}_{1}-\boldsymbol{X}^{\varepsilon, 11}(y), \quad \forall y=\left(y_{1}, y_{2}\right) \in(0,1)^{2} .
$$

It follows immediately that

$$
\begin{aligned}
\boldsymbol{A}_{\varepsilon}^{\sharp} \boldsymbol{e}\left(\boldsymbol{W}^{\varepsilon, 11}\right) & =c_{\varepsilon}^{1}\left(\boldsymbol{e}_{1} \odot \boldsymbol{e}_{1}+\frac{\lambda_{\varepsilon}^{\sharp}}{\lambda_{\varepsilon}^{\sharp}+2 \mu_{\varepsilon}^{\sharp}} \boldsymbol{e}_{2} \odot \boldsymbol{e}_{2}\right), \quad \operatorname{Div}\left(\boldsymbol{A}_{\varepsilon}^{\sharp} \boldsymbol{e}\left(\boldsymbol{W}^{\varepsilon, 11}\right)\right)=\mathbf{0}, \text { in } \mathbb{R}^{2}, \\
\boldsymbol{e}\left(\boldsymbol{W}^{\varepsilon, 11}\right) & =\frac{c_{\varepsilon}^{1}}{\lambda_{\varepsilon}^{\sharp}+2 \mu_{\varepsilon}^{\sharp}} \boldsymbol{e}_{1} \odot \boldsymbol{e}_{1} \quad \text { and } \quad \boldsymbol{A}_{*}^{\varepsilon}\left(\boldsymbol{e}_{1} \odot \boldsymbol{e}_{1}\right):\left(\boldsymbol{e}_{1} \odot \boldsymbol{e}_{1}\right)=c_{\varepsilon}^{1} .
\end{aligned}
$$

We finally define the corrector function $\boldsymbol{w}^{\varepsilon, 11}$ by

$$
\boldsymbol{w}^{\varepsilon, 11}(x):=\varepsilon \boldsymbol{W}^{\varepsilon, 11}\left(\frac{x}{\varepsilon}\right)=x_{1} \boldsymbol{e}_{1}-\varepsilon X^{\varepsilon, 11}\left(\frac{x}{\varepsilon}\right), \quad \text { a.e. } x \in \Omega .
$$

Similarly, an easy computation shows that the minimizer in the definition (2.38) of $\boldsymbol{A}_{*}^{\varepsilon}\left(\boldsymbol{e}_{1} \odot \boldsymbol{e}_{2}\right)$ : $\left(\boldsymbol{e}_{1} \odot \boldsymbol{e}_{2}\right)$ is the $Y$-periodic function $\boldsymbol{X}^{\varepsilon, 12}$ defined by

$$
\boldsymbol{X}^{\varepsilon, 12}(y):=\boldsymbol{X}^{\varepsilon, 12}\left(y_{1}\right):=\left(\int_{\frac{1}{2}}^{y_{1}}\left(1-\frac{c_{\varepsilon}^{2}}{\mu_{\varepsilon}^{\sharp}}\right) d t\right) \boldsymbol{e}_{2}, \text { a.e. } y=\left(y_{1}, y_{2}\right) \in(0,1)^{2},
$$

where

$$
c_{\varepsilon}^{2}:=\left(\int_{0}^{1} \frac{d t}{\mu_{\varepsilon}^{\sharp}}\right)^{-1} .
$$

Let us define $\boldsymbol{W}^{\varepsilon, 12}$ by

$$
\boldsymbol{W}^{\varepsilon, 12}(y):=y_{1} \boldsymbol{e}_{2}-\boldsymbol{X}^{\varepsilon, 12}(y), \quad \forall y=\left(y_{1}, y_{2}\right) \in(0,1)^{2} .
$$

It follows that

$$
\begin{gathered}
\boldsymbol{A}_{\varepsilon}^{\sharp} \boldsymbol{e}\left(\boldsymbol{W}^{\varepsilon, 12}\right)=2 c_{\varepsilon}^{2} \boldsymbol{e}_{1} \odot \boldsymbol{e}_{2}, \quad \operatorname{Div}\left(\boldsymbol{A}_{\varepsilon}^{\sharp} \boldsymbol{e}\left(\boldsymbol{W}^{\varepsilon, 12}\right)\right)=\mathbf{0}, \text { in } \quad \mathbb{R}^{2}, \\
\boldsymbol{e}\left(\boldsymbol{W}^{\varepsilon, 12}\right)=\frac{c_{\varepsilon}^{2}}{\mu_{\varepsilon}^{\sharp}} \boldsymbol{e}_{1} \odot \boldsymbol{e}_{2} \quad \text { and } \quad \boldsymbol{A}_{*}^{\varepsilon}\left(\boldsymbol{e}_{1} \odot \boldsymbol{e}_{2}\right):\left(\boldsymbol{e}_{1} \odot \boldsymbol{e}_{2}\right)=c_{\varepsilon}^{2} .
\end{gathered}
$$

We define the corrector function $\boldsymbol{w}^{\varepsilon, 12}$ by

$$
\boldsymbol{w}^{\varepsilon, 12}(x):=\varepsilon \boldsymbol{W}^{\varepsilon, 12}\left(\frac{x}{\varepsilon}\right)=x_{1} \boldsymbol{e}_{2}-\varepsilon X^{\varepsilon, 12}\left(\frac{x}{\varepsilon}\right), \quad \text { a.e. } x \in \Omega .
$$

Denote by $c_{*}^{1}$ and $c_{*}^{2}$ the constants defined by

$$
c_{*}^{1}:=\lim _{\varepsilon \rightarrow 0}\left(\int_{0}^{1} \frac{d t}{\lambda_{\varepsilon}^{\sharp}+2 \mu_{\varepsilon}^{\sharp}}\right)^{-1} \quad \text { and } \quad c_{*}^{2}:=2 \lim _{\varepsilon \rightarrow 0}\left(\int_{0}^{1} \frac{d t}{\mu_{\varepsilon}^{\sharp}}\right)^{-1} .
$$

Thanks to the explicit formulas (2.39)-(2.47) of the corrector functions associated with the elasticity problem $(2.6)$, Theorem 3 below provides an estimate of the entries $\boldsymbol{\xi}_{11}^{\varepsilon}$ and $\boldsymbol{\xi}_{12}^{\varepsilon}$ of the stress tensor $\boldsymbol{\xi}^{\varepsilon}$. Indeed, we have the following result: 
Theorem 3 Suppose (2.32) and (2.33) hold true and set

$$
b:=\lim _{\varepsilon \rightarrow 0}\left(\int_{0}^{1}\left(\psi_{\varepsilon}^{\sharp}\left(y_{1}\right)\right)^{2} \max \left(\mu_{\varepsilon}^{\sharp}\left(y_{1}\right), \lambda_{\varepsilon}^{\sharp}\left(y_{1}\right)+\mu_{\varepsilon}^{\sharp}\left(y_{1}\right)\right) d y_{1}\right) .
$$

i) Then, for any $i \in\{1,2\}$, the sequence of stress tensors $\boldsymbol{\xi}^{\varepsilon}=\boldsymbol{A}^{\varepsilon} \boldsymbol{e}\left(\boldsymbol{u}^{\varepsilon}\right)$ satisfies, for any $\varphi \in \mathcal{D}(\Omega)$, the estimate

$$
\int_{\Omega} \boldsymbol{\xi}_{1 i}^{\varepsilon} \varphi d x=c_{*}^{i} \int_{\Omega} \boldsymbol{e}(\boldsymbol{u}):\left(\boldsymbol{e}_{1} \odot \boldsymbol{e}_{i}\right) \varphi d x-\int_{\Omega} \psi_{\varepsilon}(x) \boldsymbol{\xi}^{\varepsilon}:\left(\boldsymbol{e}_{i} \otimes \nabla \varphi\right) d x+o(1),
$$

where $\boldsymbol{u}^{\varepsilon}$ is the solution of the problem (2.6) and $\boldsymbol{u}:=\left(u_{1}, 0\right)$ is the weak limit of $\boldsymbol{u}^{\varepsilon}$ in $\mathrm{H}^{1}\left(\Omega, \mathbb{R}^{2}\right)$.

ii) If $b=0$, then we have the distributional convergence

$$
\boldsymbol{\xi}_{1 i}^{\varepsilon} \rightarrow \frac{c_{*}^{i}}{2}\left(\frac{\partial u_{1}}{\partial x_{i}}+\frac{\partial u_{i}}{\partial x_{1}}\right) \quad \text { in } \quad \mathcal{D}^{\prime}(\Omega), \quad i \in\{1,2\}
$$

and $u_{1}$ is the unique solution of the limit problem

$$
\left\{\begin{array}{rllll}
-\operatorname{div}\left(\boldsymbol{A}_{1} \nabla u_{1}\right) & = & f_{1} & \text { in } & \Omega \\
u_{1} & = & 0 & \text { on } & \partial \Omega,
\end{array} \quad \text { where } \quad \boldsymbol{A}_{1}:=\left(\begin{array}{ll}
c_{*}^{1} & 0 \\
0 & \frac{c_{*}^{2}}{2}
\end{array}\right),\right.
$$

and the constants $c_{*}^{i}$ are defined by (2.48).

iii) Assume that $b \neq 0$ and that the Lamé coefficients $\left(\lambda_{\varepsilon}, \mu_{\varepsilon}\right)$ are constant functions in $F_{\varepsilon}$ satisfying

$$
\frac{\lambda_{\varepsilon}}{\mu_{\varepsilon}}=\ell>0 \quad \text { and } \quad \frac{\mu_{\varepsilon}\left(r_{\varepsilon}\right)^{3}}{\varepsilon}=\nu>0 .
$$

Then, we have the distributional convergences

$$
\boldsymbol{\xi}_{11}^{\varepsilon} \rightarrow c_{*}^{1} \frac{\partial u_{1}}{\partial x_{1}}, \quad \boldsymbol{\xi}_{12}^{\varepsilon} \rightarrow \frac{c_{*}^{2}}{2} \frac{\partial u_{1}}{\partial x_{2}}-\frac{4 b}{\ell+2} \frac{\partial^{3} u_{1}}{\partial x_{2}^{3}} \quad \text { in } \quad \mathcal{D}^{\prime}(\Omega),
$$

and $u_{1}$ is the unique solution of the fourth-order equation

$$
\left\{\begin{aligned}
-\operatorname{div}\left(\boldsymbol{A}_{1} \nabla u_{1}\right)+\frac{4 b}{\ell+2} \frac{\partial^{4} u_{1}}{\partial x_{2}^{4}} & =f_{1} \quad \text { in } \quad \Omega, \\
u_{1} & =0 \quad \text { on } \quad \partial \Omega, \\
\frac{\partial u_{1}}{\partial x_{2}} & =0 \quad \text { on } \quad(0,1) \times\{0,1\} .
\end{aligned}\right.
$$

Remark 4 These results extend those of [11] by establishing the optimality condition for the compactness of the class of equations (2.6) in the periodic case. A byproduct of this approach is the very weak convergence of two of the three entries of the stress tensor $\boldsymbol{\xi}^{\varepsilon}$ which was not given by the $\Gamma$-convergence approach used in [11]. 


\section{Proofs of the results}

\subsection{Proof of Theorem 1}

Proof of the weak convergence of $\left(\boldsymbol{u}^{\varepsilon}\right)$ to some $\boldsymbol{u}$ in $\mathrm{H}_{0}^{1}\left(\Omega, \mathbb{R}^{2}\right)$ :

Put $\boldsymbol{u}^{\varepsilon}$ as a test function in the elasticity problem (2.6). By the $\alpha$-coercivity of $\boldsymbol{A}^{\varepsilon}$, we have

$$
\alpha\left\|\boldsymbol{e}\left(\boldsymbol{u}^{\varepsilon}\right)\right\|_{\mathrm{L}^{2}\left(\Omega, \mathbb{R}_{s}^{2 \times 2}\right)}^{2} \leq\|\boldsymbol{f}\|_{\mathrm{H}^{-1}\left(\Omega, \mathbb{R}^{2}\right)}\left\|\boldsymbol{u}^{\varepsilon}\right\|_{\mathrm{L}^{2}\left(\Omega, \mathbb{R}^{2}\right)} .
$$

This, coupled with the Poincaré inequality and the Korn inequality, implies that $\left(\boldsymbol{u}^{\varepsilon}\right)$ is bounded in $\mathrm{H}_{0}^{1}\left(\Omega, \mathbb{R}^{2}\right)$. Therefore, the sequence $\left(\boldsymbol{u}^{\varepsilon}\right)$ weakly converges to some $\boldsymbol{u}$ in $\mathrm{H}_{0}^{1}\left(\Omega, \mathbb{R}^{2}\right)$.

Proof of $u_{2}=0$ :

On the interval $[0,1]$ we define the functions $v_{\varepsilon}$ and $v$ by

$$
v_{\varepsilon}\left(x_{1}\right):=\int_{0}^{1}\left|u_{2}^{\varepsilon}\left(x_{1}, x_{2}\right)\right| d x_{2} \text { and } v\left(x_{1}\right):=\int_{0}^{1}\left|u_{2}\left(x_{1}, x_{2}\right)\right| d x_{2} \quad \text { a.e. } x_{1} \in(0,1),
$$

where $\left(u_{1}, u_{2}\right):=\boldsymbol{u}$ is the limit of $\boldsymbol{u}^{\varepsilon}$. Then, $v_{\varepsilon} \in \mathrm{H}_{0}^{1}(0,1)$ and weakly converges to $v$ in $\mathrm{H}_{0}^{1}(0,1)$. Hence, the sequence of continuous functions $\left(v_{\varepsilon}\right)$ converges uniformly to $v$ in $(0,1)$. Moreover, by the Cauchy-Schwarz inequality we have

$$
\begin{aligned}
\int_{0}^{1} \mu_{\varepsilon}\left(x_{1}\right) v_{\varepsilon}\left(x_{1}\right) d x_{1} & =\int_{0}^{1} \mu_{\varepsilon}\left(x_{1}\right)\left(\int_{0}^{1}\left|u_{2}^{\varepsilon}\left(x_{1}, x_{2}\right)\right| d x_{2}\right) d x_{1} \\
& \leq \int_{0}^{1} \mu_{\varepsilon}\left(x_{1}\right)\left(\int_{0}^{1}\left|\frac{\partial u_{2}^{\varepsilon}}{\partial x_{2}}\left(x_{1}, x_{2}\right)\right| d x_{2}\right) d x_{1} \\
& =\int_{\Omega} \mu_{\varepsilon}\left(x_{1}\right)\left|\frac{\partial u_{2}^{\varepsilon}}{\partial x_{2}}\left(x_{1}, x_{2}\right)\right| d x \\
& \leq\left(\int_{0}^{1} \mu_{\varepsilon}\left(x_{1}\right) d x_{1}\right)^{\frac{1}{2}}\left(\int_{\Omega} \mu_{\varepsilon}\left(x_{1}\right)\left|\frac{\partial u_{2}^{\varepsilon}}{\partial x_{2}}\left(x_{1}, x_{2}\right)\right|^{2} d x\right)^{\frac{1}{2}} .
\end{aligned}
$$

Taking into account the boundedness of the elastic energy density $\boldsymbol{A}^{\varepsilon} \boldsymbol{e}\left(\boldsymbol{u}^{\varepsilon}\right): \boldsymbol{e}\left(\boldsymbol{u}^{\varepsilon}\right)$ in $\mathrm{L}^{1}(\Omega)$, there exists a positive constant $c$ such that

$$
\int_{0}^{1} \mu_{\varepsilon}\left(x_{1}\right) v_{\varepsilon}\left(x_{1}\right) d x_{1} \leq c \sqrt{\bar{\mu}_{\varepsilon}} .
$$

Then, since the sequence $\left(v_{\varepsilon}\right)$ uniformly converges to $v$, we obtain

$$
\begin{aligned}
\int_{0}^{1} \mu_{\varepsilon}\left(x_{1}\right) v_{\varepsilon}\left(x_{1}\right) d x_{1} & =\int_{0}^{1} \mu_{\varepsilon}\left(x_{1}\right)\left(v_{\varepsilon}-v\right)\left(x_{1}\right) d x_{1}+\int_{0}^{1} \mu_{\varepsilon}\left(x_{1}\right) v\left(x_{1}\right) d x_{1} \\
& \leq \bar{\mu}_{\varepsilon} o(1)+c \sqrt{\bar{\mu}_{\varepsilon}} .
\end{aligned}
$$

This combined with $(2.9)$ yields

$$
\int_{0}^{1} v_{\varepsilon}\left(x_{1}\right) \frac{\mu_{\varepsilon}\left(x_{1}\right)}{\bar{\mu}_{\varepsilon}} d x_{1} \leq o(1)+\frac{c}{\sqrt{\bar{\mu}_{\varepsilon}}} \underset{\varepsilon \rightarrow 0}{\longrightarrow} 0 .
$$

On the other hand, using (2.10) and the uniform convergence of the sequence $\left(v_{\varepsilon}\right)$ to $v$, estimate (3.5) gives

$$
\int_{0}^{1} v\left(x_{1}\right) \sigma\left(d x_{1}\right)=0
$$


Since $\operatorname{supp}(\sigma)=[0,1]$ and $v$ is a non-negative function on $[0,1]$, it comes that $v\left(x_{1}\right)=0$ for any $x_{1} \in[0,1]$. Therefore, in view of definition (3.1), $u_{2}(x)=0$, a.e. $x \in \Omega$.

Proof of the estimate 2.12):

Let $\varphi \in \mathcal{D}(\Omega)$. By the strong convergence (2.11), we have

$$
\begin{aligned}
o(1)=\int_{\Omega} \boldsymbol{f} \cdot \boldsymbol{e}_{i} \psi_{\varepsilon} \varphi d x & =\int_{\Omega} \boldsymbol{\xi}^{\varepsilon}: \boldsymbol{e}\left(\psi_{\varepsilon} \varphi \boldsymbol{e}_{i}\right) d x \\
& =\int_{\Omega} \frac{\partial \psi_{\varepsilon}}{\partial x_{1}} \boldsymbol{\xi}^{\varepsilon}:\left(\boldsymbol{e}_{i} \otimes \boldsymbol{e}_{1}\right) \varphi d x+\int_{\Omega} \psi_{\varepsilon} \boldsymbol{\xi}^{\varepsilon}:\left(\boldsymbol{e}_{i} \otimes \nabla \varphi\right) d x
\end{aligned}
$$

Since $\nabla \psi_{\varepsilon}=\boldsymbol{e}_{1}$ in $F_{\varepsilon}$, the last equality writes

$$
\begin{aligned}
o(1)=\int_{\Omega_{\varepsilon}} \frac{\partial \psi_{\varepsilon}}{\partial x_{1}} \boldsymbol{\xi}^{\varepsilon} & :\left(\boldsymbol{e}_{i} \otimes \boldsymbol{e}_{1}\right) \varphi d x \\
& +\int_{F_{\varepsilon}} \boldsymbol{\xi}^{\varepsilon}:\left(\boldsymbol{e}_{i} \otimes \boldsymbol{e}_{1}\right) \varphi d x+\int_{\Omega} \psi_{\varepsilon} \boldsymbol{\xi}^{\varepsilon}:\left(\boldsymbol{e}_{i} \otimes \nabla \varphi\right) d x .
\end{aligned}
$$

Moreover, $\boldsymbol{\xi}^{\varepsilon}=\boldsymbol{A} \boldsymbol{e}\left(\boldsymbol{u}^{\varepsilon}\right)$ in $\Omega^{\varepsilon}$. Then, the stress tensor $\boldsymbol{\xi}^{\varepsilon}$ is $L^{2}$-bounded in $\Omega_{\varepsilon}$, and again by 2.11 ,

$$
\int_{\Omega_{\varepsilon}} \frac{\partial \psi_{\varepsilon}}{\partial x_{1}} \boldsymbol{\xi}^{\varepsilon}:\left(\boldsymbol{e}_{i} \otimes \boldsymbol{e}_{1}\right) \varphi d x=o(1)
$$

It follows form $(3.8)$ and $(3.9)$ that

$$
o(1)=\int_{F_{\varepsilon}} \boldsymbol{\xi}^{\varepsilon}:\left(\boldsymbol{e}_{i} \otimes \boldsymbol{e}_{1}\right) \varphi d x+\int_{\Omega} \psi_{\varepsilon} \boldsymbol{\xi}^{\varepsilon}:\left(\boldsymbol{e}_{i} \otimes \nabla \varphi\right) d x .
$$

Therefore,

$$
\begin{aligned}
\int_{\Omega} \boldsymbol{\xi}^{\varepsilon}:\left(\boldsymbol{e}_{1} \otimes \boldsymbol{e}_{i}\right) \varphi d x & =\int_{\Omega_{\varepsilon}} \boldsymbol{A} \boldsymbol{e}\left(\boldsymbol{u}^{\varepsilon}\right):\left(\boldsymbol{e}_{1} \otimes \boldsymbol{e}_{i}\right) \varphi d x+\int_{F_{\varepsilon}} \boldsymbol{\xi}^{\varepsilon}:\left(\boldsymbol{e}_{1} \otimes \boldsymbol{e}_{i}\right) \varphi d x \\
& =\int_{\Omega} \boldsymbol{A} \boldsymbol{e}(\boldsymbol{u}):\left(\boldsymbol{e}_{1} \otimes \boldsymbol{e}_{i}\right) \varphi d x+\int_{F_{\varepsilon}} \boldsymbol{\xi}^{\varepsilon}:\left(\boldsymbol{e}_{1} \otimes \boldsymbol{e}_{i}\right) \varphi d x+o(1) \\
& =\int_{\Omega} \boldsymbol{A} \boldsymbol{e}(\boldsymbol{u}):\left(\boldsymbol{e}_{1} \otimes \boldsymbol{e}_{i}\right) \varphi d x-\int_{\Omega} \psi_{\varepsilon} \boldsymbol{\xi}^{\varepsilon}:\left(\boldsymbol{e}_{i} \otimes \nabla \varphi\right) d x+o(1),
\end{aligned}
$$

which implies 2.12.

Proof of (2.14):

By the Cauchy-Schwarz inequality, the boundedness of the energy density $\boldsymbol{A}^{\varepsilon} \boldsymbol{e}\left(\boldsymbol{u}^{\varepsilon}\right): \boldsymbol{e}\left(\boldsymbol{u}^{\varepsilon}\right)$ in $\mathrm{L}^{1}(\Omega)$, we have by 2.7 and $(2.8)$

$$
\begin{aligned}
& \left|\int_{\Omega} \psi_{\varepsilon}(x) \boldsymbol{\xi}^{\varepsilon}:\left(\boldsymbol{e}_{i} \otimes \nabla \varphi\right) d x\right| \\
& \quad=\left|\int_{\Omega} \boldsymbol{A}^{\varepsilon} \boldsymbol{e}\left(u^{\varepsilon}\right): \psi_{\varepsilon}(x)\left(\boldsymbol{e}_{i} \otimes \nabla \varphi\right) d x\right| \\
& \quad \leq\left(\int_{\Omega} \boldsymbol{A}^{\varepsilon} \boldsymbol{e}\left(u^{\varepsilon}\right): \boldsymbol{e}\left(u^{\varepsilon}\right) d x\right)^{\frac{1}{2}}\left(\int_{\Omega} \psi_{\varepsilon}^{2}(x) \boldsymbol{A}^{\varepsilon}\left(\boldsymbol{e}_{i} \otimes \nabla \varphi\right):\left(\boldsymbol{e}_{i} \otimes \nabla \varphi\right) d x\right)^{\frac{1}{2}} \\
& \quad \leq c\left(\int_{\Omega_{\varepsilon}} \psi_{\varepsilon}^{2}(x) \boldsymbol{A}\left(\boldsymbol{e}_{i} \otimes \nabla \varphi\right):\left(\boldsymbol{e}_{i} \otimes \nabla \varphi\right) d x+\int_{F_{\varepsilon}} \psi_{\varepsilon}^{2}(x) \boldsymbol{B}^{\varepsilon}\left(\boldsymbol{e}_{i} \otimes \nabla \varphi\right):\left(\boldsymbol{e}_{i} \otimes \nabla \varphi\right) d x\right)^{\frac{1}{2}},
\end{aligned}
$$


which, by (2.4) and the strong convergence (2.11) of $\psi_{\varepsilon}$ to zero, gives

$$
\left|\int_{\Omega} \psi_{\varepsilon}(x) \boldsymbol{\xi}^{\varepsilon}:\left(\boldsymbol{e}_{i} \otimes \nabla \varphi\right) d x\right| \leq c\left(\int_{\Omega} \psi_{\varepsilon}^{2}(x) \max \left(\mu_{\varepsilon}(x), \lambda_{\varepsilon}(x)+\mu_{\varepsilon}(x)\right) d x\right)^{\frac{1}{2}}+o(1) .
$$

Therefore, due to 2.13 we have

$$
\int_{\Omega} \psi_{\varepsilon}(x) \boldsymbol{\xi}^{\varepsilon}:\left(\boldsymbol{e}_{i} \otimes \nabla \varphi\right)=o(1)
$$

It follows from 2.12 and the definition 2.15 of $\boldsymbol{A}_{1}$ that

$$
\int_{\Omega} \boldsymbol{\xi}^{\varepsilon}:\left(\boldsymbol{e}_{i} \odot \boldsymbol{e}_{1}\right) \varphi d x=\int_{\Omega} \boldsymbol{A} \boldsymbol{e}(\boldsymbol{u}):\left(\boldsymbol{e}_{i} \odot \boldsymbol{e}_{1}\right) \varphi d x+o(1)=\int_{\Omega} \boldsymbol{A}_{1} \nabla u_{1} \cdot \boldsymbol{e}_{i} \varphi d x+o(1)
$$

which implies the distributional convergence

$$
\boldsymbol{\xi}_{1 i}^{\varepsilon}=\boldsymbol{\xi}^{\varepsilon}:\left(\boldsymbol{e}_{i} \odot \boldsymbol{e}_{1}\right) \rightarrow \boldsymbol{A}_{1} \nabla u_{1} \cdot \boldsymbol{e}_{i} \quad \text { in } \mathcal{D}^{\prime}(\Omega), \quad \forall i \in\{1,2\} .
$$

This combined with the equation

$$
-\frac{\partial}{\partial x_{1}}\left(\boldsymbol{\xi}_{11}^{\varepsilon}\right)-\frac{\partial}{\partial x_{2}}\left(\boldsymbol{\xi}_{12}^{\varepsilon}\right)=f_{1}, \quad \text { in } \Omega
$$

gives the limit problem (2.14). Moreover, for any $\boldsymbol{u}$ and $\boldsymbol{v}$ in $\mathbb{R}^{2}$, we have

$$
\boldsymbol{A}_{1} \boldsymbol{u} \cdot \boldsymbol{v}=\boldsymbol{A}\left(\boldsymbol{e}_{1} \odot \boldsymbol{u}\right):\left(\boldsymbol{e}_{1} \odot \boldsymbol{v}\right)=\left(\boldsymbol{e}_{1} \odot \boldsymbol{u}\right): \boldsymbol{A}\left(\boldsymbol{e}_{1} \odot \boldsymbol{v}\right)=\boldsymbol{A}_{1} \boldsymbol{v} \cdot \boldsymbol{u} .
$$

Then, by the positive definiteness (2.1) of $\boldsymbol{A}$, the matrix $\boldsymbol{A}_{1}$ defined by (2.15) is symmetric positive. This concludes the proof of Theorem 1 .

\subsection{Proof of Theorem 2}

Before proceeding with the proof of Theorem 2 we review the notion of two-scale convergence for thin structures. For more details we refer to [1], [25] and [11]. Throughout this section $I$ denotes the open interval $(-1,1)$.

\subsubsection{Short review on two-scale convergence for thin strips}

Let $\left(u_{\varepsilon}\right)$ be a sequence in $\mathrm{L}^{2}(\Omega)$ and $\left(\nu_{\varepsilon}\right)$ be the sequence of Radon measures defined on $\Omega \times I$ by

$$
\nu_{\varepsilon}(d x, d y):=\sum_{k \geq 1} \nu_{k}^{\varepsilon} u_{\varepsilon}(x) \frac{\mathbf{1}_{F_{k}^{\varepsilon}}(x)}{\left|F_{k}^{\varepsilon}\right|} d x \delta_{y_{k}^{\varepsilon}(x)}(d y),
$$

where $y_{k}^{\varepsilon}$ is the projection function defined by 2.19$)$. For any $\varphi \in C_{0}^{\infty}(\Omega \times I, \mathbb{R})$ we have

$$
\int_{\Omega \times I} \varphi(x, y) \nu_{\varepsilon}(d x, d y)=\sum_{k \geq 1} \nu_{k}^{\varepsilon} f_{F_{k}^{\varepsilon}} u_{\varepsilon}(x) \varphi\left(x, y_{k}^{\varepsilon}(x)\right) d x
$$


Definition 1 A sequence $\left(u_{\varepsilon}\right)$ in $\mathrm{L}^{2}(\Omega)$ is said to two-scale converge to $v \in \mathrm{L}^{2}(\Omega \times I)$ if, for any $\varphi \in \mathcal{D}(\Omega \times I)$ we have

$$
\lim _{\varepsilon \rightarrow 0}\left[\sum_{k \geq 1} \nu_{k}^{\varepsilon} f_{F_{k}^{\varepsilon}} u_{\varepsilon}(x) \varphi\left(x, y_{k}^{\varepsilon}(x)\right) d x\right]=f_{\Omega} f_{I} v(x, y) \varphi(x, y) d y d x .
$$

Then, the two-scale convergence of $\left(u_{\varepsilon}\right)$ to $v \in \mathrm{L}^{2}(\Omega \times I)$ implies, among other things, that the sequence of Radon measures $\nu_{\varepsilon}(d x, d y)$, defined by (3.11), weakly $*$ converges to a measure which is absolutely continuous with respect to the Lebesgue measure $d x d y$ with a density $v$. A sequence of vectors or tensors will be said to two-scale converge if its components two-scale converge in the sense of Definition 1 . Note that the constant sequence 1 defined on $\Omega$ two-scale converges to the constant function $\nu$ defined on $\Omega \times I$.

Following [25, 11], but with a countably infinite set of strips in the period cell, we obtain:

Lemma 1 Assume that (2.17), (2.21) and (2.22) hold true.

i) Let $\left(\boldsymbol{u}^{\varepsilon}\right)$ be a sequence in $\mathrm{L}^{2}\left(\Omega, \mathbb{R}^{2}\right)$ such that

$$
\sum_{k \geq 1} \nu_{k}^{\varepsilon} f_{F_{k}^{\varepsilon}}\left|\boldsymbol{u}^{\varepsilon}(x)\right|^{2} d x \leq c<\infty
$$

Then, up to a subsequence, $\left(\boldsymbol{u}^{\varepsilon}\right)$ two-scale converges to some $\boldsymbol{v} \in \mathrm{L}^{2}\left(\Omega \times I, \mathbb{R}^{2}\right)$.

ii) Suppose that $\left(\boldsymbol{u}^{\varepsilon}\right)$ is bounded in $\mathrm{H}^{1}\left(\Omega, \mathbb{R}^{2}\right)$. Then

$$
\sum_{k \geq 1} \nu_{k}^{\varepsilon} f_{F_{k}^{\varepsilon}}\left|\boldsymbol{u}^{\varepsilon}(x)\right|^{2} d x \leq c\left(\left\|\boldsymbol{u}^{\varepsilon}\right\|_{L^{2}\left(\Omega, \mathbb{R}^{2}\right)}^{2}+\varepsilon^{2}\left\|\nabla \boldsymbol{u}^{\varepsilon}\right\|_{L^{2}\left(\Omega, \mathbb{R}^{2 \times 2}\right)}^{2}\right) .
$$

iii) If $\left(\boldsymbol{u}^{\varepsilon}\right)$ converges strongly to some $\boldsymbol{u}$ in $\mathrm{L}^{2}\left(\Omega, \mathbb{R}^{2}\right)$ then

$$
\sum_{k \geq 1} \nu_{k}^{\varepsilon} f_{F_{k}^{\varepsilon}}\left|\boldsymbol{u}^{\varepsilon}(x)-\boldsymbol{u}(x)\right|^{2} d x=o(1) .
$$

Suppose, in addition, that $\left(\boldsymbol{u}^{\varepsilon}\right)$ two-scale converges to some $\boldsymbol{v} \in \mathrm{L}^{2}(\Omega \times I, \mathbb{R})$, then we have

$$
\boldsymbol{u}(x)=\frac{1}{\nu} f_{I} \boldsymbol{v}(x, y) d x \quad \text { a.e. } x \in \Omega
$$

iv) If the sequence $\left(\boldsymbol{u}^{\varepsilon}\right)$ has an energy density $\boldsymbol{A}^{\varepsilon} \boldsymbol{e}\left(\boldsymbol{u}^{\varepsilon}\right): \boldsymbol{e}\left(\boldsymbol{u}^{\varepsilon}\right)$ bounded in $\mathrm{L}^{1}(\Omega)$, then there exist $\boldsymbol{v} \in \mathrm{L}^{2}\left(\Omega \times I, \mathbb{R}^{2}\right), w \in \mathrm{L}^{2}(\Omega \times I, \mathbb{R})$ and $\chi \in \mathrm{L}^{2}\left(\Omega \times I, \mathbb{R}_{s}^{2 \times 2}\right)$ such that, up to a subsequence, the sequences $\left(\boldsymbol{u}^{\varepsilon}\right),\left(u_{2}^{\varepsilon} / r_{\varepsilon}\right)$ and $\left(\boldsymbol{e}\left(\boldsymbol{u}^{\varepsilon}\right) / r_{\varepsilon}\right)$ two-scale converge to $\boldsymbol{v}, w$ and $\boldsymbol{\chi}$, respectively.

Suppose, in addition, that the sequence $\left(\boldsymbol{u}^{\varepsilon}\right)$ strongly converges to some $\boldsymbol{u}$ in $\mathrm{L}^{2}\left(\Omega, \mathbb{R}^{2}\right)$, then

$$
\boldsymbol{u} \in \mathrm{H}_{0}^{1}\left(\Omega, \mathbb{R}^{2}\right), \quad \frac{\partial^{2} u_{1}}{\partial x_{2}^{2}} \in \mathrm{L}^{2}(\Omega) \quad \text { and } \quad u_{2}=0 \quad \text { a.e. in } \Omega .
$$

Moreover, there exists a function $q \in \mathrm{L}^{2}(\Omega)$ such that, up to a subsequence, the sequence $\left(\boldsymbol{e}\left(\boldsymbol{u}^{\varepsilon}\right) / r_{\varepsilon}\right)_{22}$ two-scale converges to

$$
\chi_{22}:=q(x)-\nu \frac{\partial^{2} u_{1}}{\partial x_{2}^{2}}(x) y .
$$


For the proof of Lemma 1] we refer to [25, 11] where the techniques can easily be adapted to the case of a countably infinite set of strips in the period cell. Test functions of the type $r_{\varepsilon}^{2} \varphi\left(x, y_{\varepsilon}(x)\right), r_{\varepsilon} \varphi\left(x, y_{\varepsilon}(x)\right)$ and $\varphi\left(x, y_{\varepsilon}(x)\right)$ are successively used with suitable smooth functions $\varphi$. The proof of Lemma 2 below follows the same procedure and will be detailed for the reader's convenience.

In the sequel, we shall need to compute $\chi_{11}$ and $\chi_{12}$ in terms of $\chi_{22}$ where $\chi$ is the two-scale limit of the sequence $\left(\boldsymbol{e}\left(\boldsymbol{u}^{\varepsilon}\right) / r_{\varepsilon}\right)$ in Lemma 1. This is the aim of the following result:

Lemma 2 There exist two functions $r$ and $s$ in $\mathrm{L}^{2}(\Omega)$ such that

$$
\chi_{11}(x, y)=-\left(\frac{\ell}{2+\ell}\right) \chi_{22}(x, y)+r(x) \text { and } \chi_{12}(x, y)=s(x) \text { a.e. }(x, y) \in \Omega \times I .
$$

\section{Proof of Lemma 2}

Let $\varphi \in \mathcal{D}(\Omega \times I, \mathbb{R})$. In view of 2.19) and (2.27), $\varphi\left(x, y_{\varepsilon}(x)\right)=0$ a.e. $x \in \partial F_{\varepsilon}$. Then, putting $r_{\varepsilon}^{2} \varphi\left(x, y_{\varepsilon}(x)\right) e_{1}$ as a test function in the equation 2.6 and integrating by parts on $F_{\varepsilon}$, we obtain

$$
\begin{aligned}
o(1) & =\int_{F_{\varepsilon}} \boldsymbol{\xi}^{\varepsilon}: \nabla\left(\left(r_{\varepsilon}\right)^{2} \varphi\left(x, y_{\varepsilon}(x)\right) \boldsymbol{e}_{1}\right) d x \\
& =\sum_{k \geq 1}\left(r_{k}^{\varepsilon}\right)^{2} \int_{F_{k}^{\varepsilon}}\left(2 \mu_{k}^{\varepsilon} \boldsymbol{e}\left(\boldsymbol{u}^{\varepsilon}\right)+\lambda_{k}^{\varepsilon} \operatorname{Tr}\left(\boldsymbol{e}\left(\boldsymbol{u}^{\varepsilon}\right)\right) \boldsymbol{I}_{2}\right): \nabla\left(\varphi\left(x, y_{k}^{\varepsilon}(x)\right) \boldsymbol{e}_{1}\right) d x .
\end{aligned}
$$

Moreover, by (2.19), we have

$$
\nabla\left(\varphi\left(x, y_{k}^{\varepsilon}(x)\right)\right)=\left(\frac{\partial \varphi}{\partial x_{1}}+\frac{1}{r_{k}^{\varepsilon}} \frac{\partial \varphi}{\partial y}\right) \boldsymbol{e}_{1} \odot \boldsymbol{e}_{1}+\frac{\partial \varphi}{\partial x_{2}} \boldsymbol{e}_{1} \otimes \boldsymbol{e}_{2}, \quad \forall x \in F_{\varepsilon} .
$$

Then, by $(2.21)$, since the sequence $\left(\boldsymbol{e}\left(\boldsymbol{u}^{\varepsilon}\right) / r_{\varepsilon}\right)$ two-scale converges to $\boldsymbol{\chi}$ and $\left|F_{k}^{\varepsilon}\right| \approx\left(2 r_{k}^{\varepsilon}\right) / \varepsilon$, estimate (3.14) writes

$$
\begin{aligned}
o(1)=4 \sum_{k \geq 1} \nu_{k}^{\varepsilon} f_{F_{k}^{\varepsilon}} \frac{\partial u_{1}^{\varepsilon}}{\partial x_{1}} \frac{1}{r_{k}^{\varepsilon}} \frac{\partial \varphi}{\partial y}\left(x, y_{k}^{\varepsilon}(x)\right) d x \\
+2 \ell \sum_{k \geq 1} \nu_{k}^{\varepsilon} f_{F_{k}^{\varepsilon}}\left(\frac{\partial u_{1}^{\varepsilon}}{\partial x_{1}}+\frac{\partial u_{2}^{\varepsilon}}{\partial x_{2}}\right) \frac{1}{r_{k}^{\varepsilon}} \frac{\partial \varphi}{\partial y}\left(x, y_{k}^{\varepsilon}(x)\right) d x,
\end{aligned}
$$

which, again thanks to the two-scale convergence of $\left(\boldsymbol{e}\left(\boldsymbol{u}^{\varepsilon}\right) / r_{\varepsilon}\right)$ to $\boldsymbol{\chi}$, yields

$$
f_{\Omega} f_{I}\left(4 \chi_{11}(x, y) \frac{\partial \varphi}{\partial y}(x, y)+2 \ell\left(\chi_{11}(x, y)+\chi_{22}(x, y)\right) \frac{\partial \varphi}{\partial y}(x, y)\right) d y d x=0
$$

Therefore, there exists a function $m \in \mathrm{L}^{2}(\Omega)$ such that

$$
(2+\ell) \chi_{11}(x, y)+\ell \chi_{22}(x, y)=m(x) \quad \text { a.e. }(x, y) \in \Omega \times I .
$$

It follows that

$$
\chi_{11}(x, y)=-\left(\frac{\ell}{2+\ell}\right) \chi_{22}(x, y)+r(x) \quad \text { a.e. }(x, y) \in \Omega \times I, \quad \text { where } \quad r:=\frac{m}{2+\ell} \in \mathrm{L}^{2}(\Omega) .
$$


Similarly, put $r_{\varepsilon}^{2} \varphi\left(x, y_{\varepsilon}(x)\right) e_{2}$ as a test function in the equation $(2.6)$ and integrate by parts. We obtain

$$
\sum_{k \geq 1} 2 \nu_{k}^{\varepsilon} f_{F_{k}^{\varepsilon}}\left(\frac{\partial u_{1}^{\varepsilon}}{\partial x_{2}}+\frac{\partial u_{2}^{\varepsilon}}{\partial x_{1}}\right) \frac{1}{r_{k}^{\varepsilon}} \frac{\partial \varphi}{\partial y}\left(x, y_{k}^{\varepsilon}(x)\right) d x=o(1),
$$

which, since the sequence $\left(\boldsymbol{e}\left(\boldsymbol{u}^{\varepsilon}\right) / r_{\varepsilon}\right)$ two-scale converges to $\boldsymbol{\chi}$, implies

$$
f_{\Omega} f_{I} \chi_{12}(x, y) \frac{\partial \varphi}{\partial y}(x, y) d y d x=0
$$

It follows that there exists a function $s \in \mathrm{L}^{2}(\Omega)$ such that

$$
\chi_{12}(x, y)=s(x) \quad \text { a.e. }(x, y) \in \Omega \times I .
$$

Lemma 2 is proved.

We are now in a position to prove Theorem 2 .

\subsubsection{Proof of Theorem 2}

For any $\varphi \in \mathcal{D}(\Omega)$, since $\psi_{\varepsilon}\left\|\boldsymbol{\xi}^{\varepsilon}\right\| \mathbf{1}_{\Omega_{\varepsilon}}$ strongly converges to zero in $\mathrm{L}^{1}(\Omega)$, by (2.26) and $(2.27)$ we have the following estimates:

$$
\begin{aligned}
\int_{\Omega} \psi_{\varepsilon}(x) \boldsymbol{\xi}^{\varepsilon}:\left(e_{i} \otimes \nabla \varphi\right) d x & =\int_{F_{\varepsilon}} \psi_{\varepsilon}(x) \boldsymbol{\xi}^{\varepsilon}:\left(e_{i} \otimes \nabla \varphi\right) d x+o(1) \\
& =\int_{F_{\varepsilon}} r_{\varepsilon}(x) y_{\varepsilon}(x) \boldsymbol{\xi}^{\varepsilon}:\left(e_{i} \otimes \nabla \varphi\right) d x+o(1) \\
& =\sum_{k \geq 1} \int_{F_{k}^{\varepsilon}} r_{k}^{\varepsilon} y_{k}^{\varepsilon}(x) \boldsymbol{\xi}^{\varepsilon}:\left(e_{i} \otimes \nabla \varphi\right) d x+o(1) \\
& =\sum_{k \geq 1} \int_{F_{k}^{\varepsilon}} r_{k}^{\varepsilon} y_{k}^{\varepsilon}(x)\left(2 \mu_{k}^{\varepsilon} \boldsymbol{e}\left(\boldsymbol{u}^{\varepsilon}\right)+\lambda_{k}^{\varepsilon} \operatorname{Tr}\left(\boldsymbol{e}\left(\boldsymbol{u}^{\varepsilon}\right)\right) \boldsymbol{I}_{2}\right):\left(e_{i} \otimes \nabla \varphi\right) d x+o(1)
\end{aligned}
$$

which, in view of (2.21), yields

$$
\begin{aligned}
\int_{\Omega} \psi_{\varepsilon}(x) \boldsymbol{\xi}^{\varepsilon}:\left(e_{i} \otimes \nabla \varphi\right) d x & =4 \sum_{k \geq 1} \nu_{k}^{\varepsilon} f_{F_{k}^{\varepsilon}} y_{k}^{\varepsilon}(x) \nabla\left(\varphi(x) e_{i}\right):\left(\frac{\boldsymbol{e}\left(\boldsymbol{u}^{\varepsilon}\right)}{r_{k}^{\varepsilon}}\right) d x \\
& +2 \ell \sum_{k \geq 1} \nu_{k}^{\varepsilon} f_{F_{k}^{\varepsilon}}\left(\frac{\partial u_{1}^{\varepsilon}}{\partial x_{1}}+\frac{\partial u_{2}^{\varepsilon}}{\partial x_{2}}\right) \frac{1}{r_{k}^{\varepsilon}} \frac{\partial \varphi}{\partial x_{i}} y_{k}^{\varepsilon}(x) d x+o(1) .
\end{aligned}
$$

It follows from $(3.15)$ and the two-scale convergence of the sequence $\left(\boldsymbol{e}\left(\boldsymbol{u}^{\varepsilon}\right) / r_{\varepsilon}\right)$ to $\boldsymbol{\chi}$, that

$$
\begin{aligned}
\int_{\Omega} \psi_{\varepsilon}(x) \boldsymbol{\xi}^{\varepsilon}:\left(e_{i} \otimes \nabla \varphi\right) d x= & 4 f_{\Omega} f_{I} y \nabla\left(\varphi(x) e_{i}\right): \chi(x, y) d y d x \\
& +2 \ell f_{\Omega} f_{I}\left(\chi_{11}+\chi_{22}\right) \frac{\partial \varphi}{\partial x_{i}} y d y d x+o(1)
\end{aligned}
$$

Using (3.13), we have

$$
\begin{aligned}
\int_{\Omega} \psi_{\varepsilon}(x) \boldsymbol{\xi}^{\varepsilon}:\left(e_{i} \otimes \nabla \varphi\right) d x=4 f_{\Omega} f_{I}\left(\chi_{1 i} \frac{\partial \varphi}{\partial x_{1}}+\chi_{i 2} \frac{\partial \varphi}{\partial x_{2}}\right) y d y d x \\
+\frac{4 \ell}{\ell+2} f_{\Omega} f_{I} \chi_{22} \frac{\partial \varphi}{\partial x_{i}} y d y d x+o(1) .
\end{aligned}
$$


Therefore, due to 3.12 and 3.13 , we obtain for $i=1$

$$
\int_{\Omega} \psi_{\varepsilon}(x) \boldsymbol{\xi}^{\varepsilon}:\left(e_{1} \otimes \nabla \varphi\right) d x=o(1)
$$

while for $i=2$

$$
\begin{aligned}
\int_{\Omega} \psi_{\varepsilon}(x) \boldsymbol{\xi}^{\varepsilon}:\left(e_{2} \otimes \nabla \varphi\right) d x & =8\left(\frac{\ell+1}{\ell+2}\right) f_{\Omega} f_{I} \chi_{22} \frac{\partial \varphi}{\partial x_{2}} y d y d x+o(1) \\
& =4 \nu\left(\frac{\ell+1}{\ell+2}\right) \int_{\Omega} \int_{I}\left(-\frac{\partial^{2} u_{1}}{\partial x_{2}^{2}} y^{2}\right) d y \frac{\partial \varphi}{\partial x_{2}} d x+o(1) \\
& =-\frac{8}{3} \nu\left(\frac{\ell+1}{\ell+2}\right) \int_{\Omega} \frac{\partial^{2} u_{1}}{\partial x_{2}^{2}} \frac{\partial \varphi}{\partial x_{2}} d x+o(1),
\end{aligned}
$$

which proves 2.28.

Now, putting $\varphi$ as a test function in the first equation of 2.6 we obtain

$$
\int_{\Omega} \boldsymbol{\xi}_{11}^{\varepsilon} \frac{\partial \varphi}{\partial x_{1}} d x+\int_{\Omega} \boldsymbol{\xi}_{12}^{\varepsilon} \frac{\partial \varphi}{\partial x_{2}} d x=\int_{\Omega} f_{1} \varphi d x
$$

which, combined with 2.12 and replacing $\varphi$ by $\partial_{2} \varphi$ in 2.28 , gives the variational formulation

$$
(\lambda+\mu) \int_{\Omega} \frac{\partial u_{1}}{\partial x_{1}} \frac{\partial \varphi}{\partial x_{1}} d x+\mu \int_{\Omega} \nabla u_{1} \nabla \varphi d x+\eta \int_{\Omega} \frac{\partial^{2} u_{1}}{\partial x_{2}^{2}} \frac{\partial^{2} \varphi}{\partial x_{2}^{2}} d x=\int_{\Omega} f_{1} \varphi d x,
$$

where $\eta$ is defined by 2.29 . This formulation implies the limit equation in 2.30 and ensures the uniqueness of the solution of the problem 2.30 using an integration by parts. Theorem 2 is then proved.

\subsection{Proof of Theorem 3}

Proof of part (i):

The reinforcing zone $F_{\varepsilon}$ is composed of strips $F_{k}^{\varepsilon}$ which are parallel to the $x_{2}$-axis and the Lamé coefficient $\mu_{\varepsilon}$ of which is not $\mathrm{L}^{1}(\Omega)$-bounded. Owing to Theorem 1, the solution $\boldsymbol{u}^{\varepsilon}$ of the elasticity problem (2.6) weakly converges in $\mathrm{H}^{1}\left(\Omega, \mathbb{R}^{2}\right)$ to some $\boldsymbol{u}=\left(u_{1}, 0\right)$. The sequel of the proof of $(i)$ is divided in two steps as follows: we first prove that the stress tensor $\boldsymbol{\xi}^{\varepsilon}$ satisfies the estimate

$$
\int_{\Omega} \boldsymbol{\xi}^{\varepsilon}:\left(\boldsymbol{e}_{1} \odot \boldsymbol{e}_{i}\right) \varphi d x=\int_{\Omega} \boldsymbol{\xi}^{\varepsilon}: \boldsymbol{e}\left(\boldsymbol{w}^{\varepsilon, 1 i}\right) \varphi d x-\int_{\Omega} \psi_{\varepsilon}(x) \boldsymbol{\xi}^{\varepsilon}:\left(\boldsymbol{e}_{i} \otimes \nabla \varphi\right) d x+o(1),
$$

for any $\varphi \in \mathcal{D}(\Omega)$, where $\boldsymbol{w}^{\varepsilon, 11}$ and $\boldsymbol{w}^{\varepsilon, 12}$ are the corrector functions defined by (2.43) and (2.47). The second step is devoted to the proof of estimate 2.50.

First step: Proof of estimate 3.18

Let $\varphi \in \mathcal{D}(\Omega)$. In view of the definition 2.43 of $\boldsymbol{w}^{\varepsilon, 11}$, putting $\boldsymbol{w}^{\varepsilon, 11} \varphi$ as a test function in 2.6 and integrating by parts, we obtain

$$
\begin{aligned}
\int_{\Omega} \boldsymbol{\xi}^{\varepsilon}: \boldsymbol{e}\left(\boldsymbol{w}^{\varepsilon, 11}\right) \varphi= & \int_{\Omega} \boldsymbol{f} \cdot \boldsymbol{w}^{\varepsilon, 11} \varphi d x-\int_{\Omega} \boldsymbol{\xi}^{\varepsilon}:\left(\boldsymbol{w}^{\varepsilon, 11} \otimes \nabla \varphi\right) d x \\
= & \int_{\Omega}\left(\boldsymbol{f} \cdot \boldsymbol{e}_{1}\right) x_{1} \varphi d x-\varepsilon \int_{\Omega} \boldsymbol{X}^{\varepsilon, 11}\left(\frac{x}{\varepsilon}\right) \cdot \boldsymbol{f}(x) \varphi d x \\
& +\varepsilon \int_{\Omega} X_{1}^{\varepsilon, 11}\left(\frac{x}{\varepsilon}\right) \boldsymbol{\xi}^{\varepsilon}:\left(\boldsymbol{e}_{1} \otimes \nabla \varphi\right) d x-\int_{\Omega} x_{1} \boldsymbol{\xi}^{\varepsilon}:\left(\boldsymbol{e}_{1} \otimes \nabla \varphi\right) d x
\end{aligned}
$$


Since $\boldsymbol{X}^{\varepsilon, 11}$ is uniformly bounded in $\Omega$, we have, again using (2.6)

$$
\begin{array}{r}
\int_{\Omega} \boldsymbol{\xi}^{\varepsilon}: \boldsymbol{e}\left(\boldsymbol{w}^{\varepsilon, 11}\right) \varphi d x=\int_{\Omega} \boldsymbol{\xi}^{\varepsilon}: \nabla\left(\varphi(x) x_{1} \boldsymbol{e}_{1}\right) d x-\int_{\Omega} \boldsymbol{\xi}^{\varepsilon}:\left(x_{1} \boldsymbol{e}_{1} \otimes \nabla \varphi\right) d x \\
+\varepsilon \int_{\Omega} X_{1}^{\varepsilon, 11}\left(\frac{x}{\varepsilon}\right) \boldsymbol{\xi}^{\varepsilon}:\left(\boldsymbol{e}_{1} \otimes \nabla \varphi\right) d x+o(1) \\
=\int_{\Omega} \boldsymbol{\xi}^{\varepsilon}:\left(\boldsymbol{e}_{1} \odot \boldsymbol{e}_{1}\right) \varphi d x+\varepsilon \int_{\Omega} X_{1}^{\varepsilon, 11}\left(\frac{x}{\varepsilon}\right) \boldsymbol{\xi}^{\varepsilon}:\left(\boldsymbol{e}_{1} \otimes \nabla \varphi\right) d x+o(1) .
\end{array}
$$

Since $\left|\boldsymbol{X}^{\varepsilon, 11}\right|$ and $\left\|\boldsymbol{\xi}^{\varepsilon}\right\|$ are bounded in $L^{2}\left(\Omega_{\varepsilon}\right)$, we have

$$
\varepsilon \int_{\Omega_{\varepsilon}} X_{1}^{\varepsilon, 11}\left(\frac{x}{\varepsilon}\right) \boldsymbol{\xi}^{\varepsilon}:\left(\boldsymbol{e}_{1} \otimes \nabla \varphi\right) d x=o(1) .
$$

Let us now estimate

$$
\varepsilon \int_{F_{\varepsilon}} X_{1}^{\varepsilon, 11}\left(\frac{x}{\varepsilon}\right) \boldsymbol{\xi}^{\varepsilon}:\left(\boldsymbol{e}_{1} \otimes \nabla \varphi\right) d x
$$

By the Cauchy-Schwarz inequality we have

$$
\begin{aligned}
& \left|\int_{F_{\varepsilon}}\left(\varepsilon X_{1}^{\varepsilon, 11}\left(\frac{x}{\varepsilon}\right)-\psi_{\varepsilon}(x)\right) \boldsymbol{\xi}^{\varepsilon}:\left(\boldsymbol{e}_{1} \otimes \nabla \varphi\right) d x\right| \\
& =\left|\int_{F_{\varepsilon}}\left(\varepsilon X_{1}^{\varepsilon, 11}\left(\frac{x}{\varepsilon}\right)-\psi_{\varepsilon}^{\sharp}\left(\frac{x_{1}}{\varepsilon}\right)\right) \boldsymbol{\xi}^{\varepsilon}:\left(\boldsymbol{e}_{1} \otimes \nabla \varphi\right) d x\right| \\
& =\varepsilon\left|\int_{F_{\varepsilon}}\left(X_{1}^{\varepsilon, 11}\left(\frac{x}{\varepsilon}\right)-\frac{1}{\varepsilon} \psi_{\varepsilon}^{\sharp}\left(\frac{x_{1}}{\varepsilon}\right)\right) \boldsymbol{A}^{\varepsilon} \boldsymbol{e}\left(\boldsymbol{u}^{\varepsilon}\right):\left(\boldsymbol{e}_{1} \otimes \nabla \varphi\right) d x\right| \\
& \leq \varepsilon\left(\int_{F_{\varepsilon}}\left(X_{1}^{\varepsilon, 11}\left(\frac{x}{\varepsilon}\right)-\frac{1}{\varepsilon} \psi_{\varepsilon}^{\sharp}\left(\frac{x_{1}}{\varepsilon}\right)\right)^{2} \boldsymbol{A}^{\varepsilon}\left(\boldsymbol{e}_{1} \otimes \nabla \varphi\right):\left(\boldsymbol{e}_{1} \otimes \nabla \varphi\right)\right)^{\frac{1}{2}} \\
& \times\left(\int_{\Omega} \boldsymbol{A}^{\varepsilon} \boldsymbol{e}\left(\boldsymbol{u}^{\varepsilon}\right): \boldsymbol{e}\left(\boldsymbol{u}^{\varepsilon}\right)\right)^{\frac{1}{2}} .
\end{aligned}
$$

Moreover, by the formula (2.35) and (2.39), we have

$$
\begin{aligned}
\left|X_{1}^{\varepsilon, 11}(y)-\frac{1}{\varepsilon} \psi_{\varepsilon}^{\sharp}\left(y_{1}\right)\right| & =\left|c_{\varepsilon}^{1} \int_{y_{1}}^{\frac{1}{2}} \frac{d t}{\lambda_{\varepsilon}^{\sharp}+2 \mu_{\varepsilon}^{\sharp}}\right|, \quad \text { a.e. } y=\left(y_{1}, y_{2}\right) \in I_{\varepsilon} \times(0,1), \\
& \leq c_{\varepsilon}^{1} \int_{I_{\varepsilon}} \frac{d t}{\lambda_{\varepsilon}^{\sharp}+2 \mu_{\varepsilon}^{\sharp}}, \quad \text { a.e. } y=\left(y_{1}, y_{2}\right) \in I_{\varepsilon} \times(0,1) .
\end{aligned}
$$

Then, due to (2.4), the periodicity of $\boldsymbol{A}^{\varepsilon}$ and the boundedness of the energy density $\boldsymbol{A}^{\varepsilon} \boldsymbol{e}\left(\boldsymbol{u}^{\varepsilon}\right)$ : $\boldsymbol{e}\left(\boldsymbol{u}^{\varepsilon}\right)$ in $\mathrm{L}^{1}(\Omega)$, there exists a positive constant $c(\varphi)$ such that

$$
\begin{aligned}
& \left|\int_{F_{\varepsilon}}\left(\varepsilon X_{1}^{\varepsilon, 11}\left(\frac{x_{1}}{\varepsilon}\right)-\psi_{\varepsilon}(x)\right) \boldsymbol{\xi}^{\varepsilon}:\left(\boldsymbol{e}_{1} \otimes \nabla \varphi\right) d x\right| \\
& \quad \leq c(\varphi) \varepsilon\left(\left(\int_{I_{\varepsilon}} \max \left(\mu_{\varepsilon}^{\sharp}, \lambda_{\varepsilon}^{\sharp}+\mu_{\varepsilon}^{\sharp}\right) d y_{1}\right)\left(\int_{I_{\varepsilon}} \frac{d y_{1}}{\lambda_{\varepsilon}^{\sharp}+2 \mu_{\varepsilon}^{\sharp}}\right)^{2}\right)^{\frac{1}{2}} .
\end{aligned}
$$

Thanks to Assumption (2.33) with $\lambda_{\varepsilon}^{\sharp}+2 \mu_{\varepsilon}^{\sharp} \geq \mu_{\varepsilon}^{\sharp}$ a.e. in $\Omega$, the left-hand side of the inequality (3.22) tends to zero, as $\varepsilon$ goes to zero. Hence,

$$
\varepsilon \int_{F_{\varepsilon}} X_{1}^{\varepsilon, 11}\left(\frac{x}{\varepsilon}\right) \boldsymbol{\xi}^{\varepsilon}:\left(\boldsymbol{e}_{1} \otimes \nabla \varphi\right) d x=\int_{F_{\varepsilon}} \psi_{\varepsilon}(x) \boldsymbol{\xi}^{\varepsilon}:\left(\boldsymbol{e}_{1} \otimes \nabla \varphi\right) d x+o(1) .
$$


Finally, putting together the estimates 3.19 , 3.20 and 3.23 we have

$$
\int_{\Omega} \boldsymbol{\xi}^{\varepsilon}: \boldsymbol{e}\left(\boldsymbol{w}^{\varepsilon, 11}\right) \varphi d x=\int_{\Omega} \boldsymbol{\xi}^{\varepsilon}:\left(\boldsymbol{e}_{1} \odot \boldsymbol{e}_{1}\right) \varphi d x+\int_{\Omega} \psi_{\varepsilon}(x) \boldsymbol{\xi}^{\varepsilon}:\left(\boldsymbol{e}_{1} \otimes \nabla \varphi\right) d x+o(1)
$$

which proves 3.18 in the case $i=1$.

Similarly, using the definition 2.47) of $\boldsymbol{w}^{\varepsilon, 12}$ and condition 2.33), we obtain

$$
\int_{\Omega} \boldsymbol{\xi}^{\varepsilon}: \boldsymbol{e}\left(\boldsymbol{w}^{\varepsilon, 12}\right) \varphi d x=\int_{\Omega} \boldsymbol{\xi}^{\varepsilon}:\left(\boldsymbol{e}_{1} \odot \boldsymbol{e}_{2}\right) \varphi d x+\int_{\Omega} \psi_{\varepsilon}(x) \boldsymbol{\xi}^{\varepsilon}:\left(\boldsymbol{e}_{2} \otimes \nabla \varphi\right) d x+o(1),
$$

which proves $(3.18)$ in the case $i=2$. This concludes the proof of the first step.

Second step: Proof of estimate 2.50)

In the case $i=1$, using $(2.42)$ and 2.43 we have

$$
\begin{aligned}
\int_{\Omega} \boldsymbol{\xi}^{\varepsilon}: \boldsymbol{e}\left(\boldsymbol{w}^{\varepsilon, 11}\right) \varphi d x & =\int_{\Omega}\left[\left(2 \mu_{\varepsilon}(x) \boldsymbol{e}\left(\boldsymbol{u}^{\varepsilon}\right)+\lambda_{\varepsilon}(x) \operatorname{Tr}\left(\boldsymbol{e}\left(\boldsymbol{u}^{\varepsilon}\right)\right) \boldsymbol{I}_{2}\right): \frac{c_{\varepsilon}^{1}\left(\boldsymbol{e}_{1} \odot \boldsymbol{e}_{1}\right)}{\lambda_{\varepsilon}(x)+2 \mu_{\varepsilon}(x)}\right] \varphi d x \\
& =\int_{\Omega} c_{\varepsilon}^{1}\left(\frac{\partial u_{1}^{\varepsilon}}{\partial x_{1}}+\frac{\lambda_{\varepsilon}(x)}{\lambda_{\varepsilon}(x)+2 \mu_{\varepsilon}(x)} \frac{\partial u_{2}^{\varepsilon}}{\partial x_{2}}\right) \varphi d x
\end{aligned}
$$

Since the sequence $\boldsymbol{u}^{\varepsilon}$ weakly converges to $\boldsymbol{u}=\left(u_{1}, 0\right)$ in $\mathrm{H}^{1}\left(\Omega, \mathbb{R}^{2}\right)$ and then strongly in $\mathrm{L}^{2}\left(\Omega, \mathbb{R}^{2}\right)$, since $c_{\varepsilon}^{1}$ strongly converges in $\mathrm{L}^{2}(\Omega)$ to the constant $c_{*}^{1}$ defined by $(2.48)$, and since the function $c_{\varepsilon}^{1} \lambda_{\varepsilon} /\left(\lambda_{\varepsilon}+2 \mu_{\varepsilon}\right)$ does not depend on the variable $x_{2}$, we have

$$
\lim _{\varepsilon \rightarrow 0}\left(\int_{\Omega} c_{\varepsilon}^{1} \frac{\partial u_{1}^{\varepsilon}}{\partial x_{1}} \varphi d x\right)=c_{*}^{1} \int_{\Omega} \frac{\partial u_{1}}{\partial x_{1}} \varphi d x
$$

and

$$
\lim _{\varepsilon \rightarrow 0}\left(\int_{\Omega} c_{\varepsilon}^{1} \frac{\lambda_{\varepsilon}(x)}{\lambda_{\varepsilon}(x)+2 \mu_{\varepsilon}(x)} \frac{\partial u_{2}^{\varepsilon}}{\partial x_{2}} \varphi d x\right)=0 .
$$

It follows from 3.24$)-(3.26)$ and the fact that $u_{2}=0$ a.e. in $\Omega$, that

$$
\begin{aligned}
\int_{\Omega} \boldsymbol{\xi}^{\varepsilon}: \boldsymbol{e}\left(\boldsymbol{w}^{\varepsilon, 11}\right) \varphi d x & =c_{*}^{1} \int_{\Omega} \frac{\partial u_{1}}{\partial x_{1}} \varphi d x+o(1) \\
& =c_{*}^{1} \int_{\Omega} \boldsymbol{e}(\boldsymbol{u}):\left(\boldsymbol{e}_{1} \odot \boldsymbol{e}_{1}\right) \varphi d x+o(1)
\end{aligned}
$$

which, coupled with estimate 3.18 proves 2.50 in the case $i=1$.

Similarly, in the case $i=2$, using 2.46$)-2.48$ we have

$$
\begin{aligned}
\int_{\Omega} \boldsymbol{\xi}^{\varepsilon}: \boldsymbol{e}\left(\boldsymbol{w}^{\varepsilon, 12}\right) \varphi d x & =\int_{\Omega}\left(\left(2 \mu_{\varepsilon}(x) \boldsymbol{e}\left(\boldsymbol{u}^{\varepsilon}\right)+\lambda_{\varepsilon}(x) \operatorname{Tr}\left(\boldsymbol{e}\left(\boldsymbol{u}^{\varepsilon}\right)\right) \boldsymbol{I}_{2}\right): \frac{c_{\varepsilon}^{2}}{\mu_{\varepsilon}(x)} \boldsymbol{e}_{1} \odot \boldsymbol{e}_{2}\right) \varphi d x \\
& =\int_{\Omega} c_{\varepsilon}^{2}\left(\frac{\partial u_{1}^{\varepsilon}}{\partial x_{2}}+\frac{\partial u_{2}^{\varepsilon}}{\partial x_{1}}\right) \varphi d x \\
& =c_{*}^{2} \int_{\Omega} \boldsymbol{e}(\boldsymbol{u}):\left(\boldsymbol{e}_{1} \odot \boldsymbol{e}_{2}\right) \varphi d x+o(1) .
\end{aligned}
$$


Putting together 3.18 and 3.27 we obtain the estimate 2.50 when $i=2$.

Proof of part (ii):

By the Cauchy-Schwarz inequality and the boundedness of the elastic energy density $\boldsymbol{A}^{\varepsilon}(x) \boldsymbol{e}\left(\boldsymbol{u}^{\varepsilon}\right)$ : $\boldsymbol{e}\left(\boldsymbol{u}^{\varepsilon}\right)$ in $\mathrm{L}^{1}(\Omega)$, there exists a positive constant $c$ such that

$$
\begin{gathered}
\left|\int_{\Omega} \psi_{\varepsilon}(x) \boldsymbol{\xi}^{\varepsilon}:\left(\boldsymbol{e}_{i} \otimes \nabla \varphi\right) d x\right|=\left|\int_{\Omega} \boldsymbol{A}^{\varepsilon} \boldsymbol{e}\left(\boldsymbol{u}^{\varepsilon}\right): \psi_{\varepsilon}(x)\left(\boldsymbol{e}_{i} \otimes \nabla \varphi\right) d x\right| \\
\leq\left(\int_{\Omega} \boldsymbol{A}^{\varepsilon} \boldsymbol{e}\left(\boldsymbol{u}^{\varepsilon}\right): \boldsymbol{e}\left(\boldsymbol{u}^{\varepsilon}\right) d x\right)^{\frac{1}{2}} \times\left(\int_{\Omega} \psi_{\varepsilon}^{2}(x) \boldsymbol{A}^{\varepsilon}\left(\boldsymbol{e}_{i} \otimes \nabla \varphi\right):\left(\boldsymbol{e}_{i} \otimes \nabla \varphi\right) d x\right)^{\frac{1}{2}} \\
\leq c\left(\int_{\Omega} \psi_{\varepsilon}^{2}(x) \boldsymbol{A}^{\varepsilon}\left(\boldsymbol{e}_{i} \otimes \nabla \varphi\right):\left(\boldsymbol{e}_{i} \otimes \nabla \varphi\right) d x\right)^{\frac{1}{2}} .
\end{gathered}
$$

Using 2.4 and the periodicity of $\psi_{\varepsilon}^{\sharp}, \lambda_{\varepsilon}^{\sharp}$ and $\mu_{\varepsilon}^{\sharp}$, we obtain from 3.28

$$
\begin{aligned}
\left|\int_{\Omega} \psi_{\varepsilon}(x) \boldsymbol{\xi}^{\varepsilon}:\left(\boldsymbol{e}_{i} \otimes \nabla \varphi\right) d x\right| & \\
\leq c & \left(\int_{\Omega}\left(\psi_{\varepsilon}(x)\right)^{2} \max \left(\mu_{\varepsilon}(x), \lambda_{\varepsilon}(x)+\mu_{\varepsilon}(x)\right) d x\right)^{\frac{1}{2}} \\
\leq c & \left(\int_{0}^{1}\left(\psi_{\varepsilon}^{\sharp}\left(y_{1}\right)\right)^{2} \max \left(\mu_{\varepsilon}^{\sharp}\left(y_{1}\right), \lambda_{\varepsilon}^{\sharp}\left(y_{1}\right)+\mu_{\varepsilon}^{\sharp}\left(y_{1}\right)\right) d y_{1}\right)^{\frac{1}{2}} .
\end{aligned}
$$

Taking (2.49) into account, the left-hand side of 3.29 tends to zero, as $\varepsilon$ goes to zero. Therefore,

$$
\lim _{\varepsilon \rightarrow 0}\left(\int_{\Omega} \psi_{\varepsilon}(x) \boldsymbol{\xi}^{\varepsilon}:\left(\boldsymbol{e}_{i} \otimes \nabla \varphi\right) d x\right)=0,
$$

which, combined with the estimate 2.50, gives the distributional convergence 2.51) and the limit problem (2.52). Then (ii) is proved.

Proof of part (iii):

If $b \neq 0$ and 2.53 is satisfied, then the convergence 2.28 of Theorem 2 implies that

$$
\lim _{\varepsilon \rightarrow 0}\left(\int_{\Omega} \psi_{\varepsilon}(x) \boldsymbol{\xi}^{\varepsilon}:\left(\boldsymbol{e}_{i} \otimes \nabla \varphi\right) d x\right)= \begin{cases}0 & \text { if } \quad i=1, \\ -\frac{4 b}{\ell+2} \int_{\Omega} \frac{\partial^{2} u_{1}}{\partial x_{2}^{2}} \frac{\partial \varphi}{\partial x_{2}}(x) d x & \text { if } \quad i=2,\end{cases}
$$

which, combined with 2.50) gives the distributional convergences (2.54). The proof of (2.55) is similar to that of 2.30 . Theorem 3 is proved.

\section{References}

[1] G. Allaire, Shape optimization by the homogenization method, Applied Mathematical sciences, 146, Springer-Verlag, New York (2001). 
[2] M. Bellieud \& G. Bouchitté, Homogenization of elliptic problems in a fiber reinforced structure. Nonlocal effects, Ann. Scuola Norm. Sup. Pisa Cl. Sci., 26 (4), 407-436 (1998).

[3] M. Bellieud \& G. Bouchitté, Homogenization of a soft elastic material reinforced by fibers, Asymptot. Anal. 32 (2), 153-183 (2002).

[4] M. Bellieud \& I. Gruais, Homogenization of an elastic material reinforced by very stiff or heavy fibers. Non-local effects. Memory effects, J. Math. Pures Appl., (9) 84 (1), 55-96 (2005).

[5] A. Bensoussan, J. L. Lions \& G. Papanicolaou, Asymptotic Analysis for Periodic Structures, North-Holland (1978).

[6] A. Beurling \& J. Deny, Espaces de Dirichlet, Acta Matematica, 99, 203-224 (1958).

[7] M. BRIAne, Homogenization in some weakly connected materials, Ricerche di Matematica, 47 (1), 51-94 (1998).

[8] M. Briane, Homogenization of high-conductivity periodic problems: Application to a general distribution of one-directional fibers, SIAM J. Math. Anal., 35 (1), 33-60 (2003).

[9] M. Briane, Homogenization of non uniformly bounded operators: critical barrier for nonlocal effects, Arch. Rat. Mech. Anal., 164, 73-101(2002).

[10] M. Briane, Nonlocal effects in two-dimensional conductivity, Arch. Rat. Mech. Anal. 182 (2), 255-267 (2006).

[11] M. Briane \& M. Camar-Eddine, Homogenization of two-dimensional elasticity problems with very stiff coefficients, J. Math. Pures et Appl., 88, 483-505 (2007).

[12] M. Briane \& J. Casado-Díaz, Two-dimensional div-curl results. Application to the lack of nonlocal effects in homogenization, Com. Part. Diff. Equa., 32, 935-969 (2007).

[13] M. Briane \& J. Casado-Díaz, Asymptotic behaviour of equicoercive diffusion energies in dimension two, Calc. Var. PDE's, 29 (4), 455-479 (2007).

[14] M. Briane \& N. Tchou, Fibered microstructures for some nonlocal Dirichlet forms, Ann. Scuola Norm. Sup. Pisa Cl. Sci., 30 (4), 681-711 (2001).

[15] G. Buttazzo, G. Dal Maso, Г-limits of integral functionals, J. Analyse Math., 37, 145-185 (1980).

[16] M. Camar-Eddine \& P. Seppecher, Closure of the set of diffusion functionals with respect to the Mosco-convergence, Math. Models Methods Appl. Sci., 12 (8), 1153-1176 (2002).

[17] M. Camar-Eddine \& P. Seppecher, Determination of the closure of the set of elasticity functionals, Arch. Rat. Mech. Anal., 170 (3), 211-245 (2003).

[18] V.N. Fenchenko \& E.Ya. Khruslov, Asymptotic of solution of differential equations with strongly oscillating matrix of coefficients which does not satisfy the condition of uniform boundedness, Dokl. AN Ukr.SSR, 4 (1981).

[19] G. Francfort \& F. Murat, Homogenization and Optimal Bounds in Linear Elasticity, Arch. Rat. Mech. Anal., 94, 307-334 (1986). 
[20] E.YA. Khruslov, Homogenized models of composite media, Composite Media and Homogenization Theory, ed. by G. Dal Maso and G.F. Dell'Antonio, in Progress in Nonlinear Differential Equations and Their Applications, Birkhaüser, 159-182 (1991).

[21] E.Ya. Khruslov \& V.A. Marchenko, Homogenization of Partial Differential Equations, Progress in Mathematical Physics, 46, Birkhäuser, Boston (2006).

[22] U. Mosco, Composite media and asymptotic Dirichlet forms, J. of Functional Analysis, 123 (2), 368-421 (1994).

[23] F. Murat, Compacité par compensation, Ann. Sc. Norm. Sup. Pisa, 5, 489-507 (1978).

[24] F. MurAt, H-convergence, Séminaire d'Analyse Fonctionnelle et Numérique, 1977-78, Université d'Alger, multicopied, 34 pp. English translation : F. MurAT \& L. TARTAR, H-convergence, Topics in the Mathematical Modelling of Composite Materials, ed. by A.V. Cherkaev \& R.V. Kohn, Progress in Nonlinear Differential Equations and their Applications, 31, Birkaüser, Boston, 21-43 (1998).

[25] C. Pideri \& P. Seppecher, A second gradient material resulting from the homogenization of an heterogeneous linear elastic medium, Continuum Mech. and Thermodyn., 9 (5), 241-257 (1997).

[26] S. Spagnolo, Sulla convergenza di soluzioni di equazioni paraboliche ed ellittiche, Ann. Scuola Norm. Sup. Pisa Cl. Sci., 22, 571-597 (1968).

[27] L. Tartar, Cours Peccot, Collège de France (1977), partly written in [24]. 